\title{
UNCONDITIONALLY OPTIMAL ERROR ESTIMATES OF THE BILINEAR-CONSTANT SCHEME FOR TIME-DEPENDENT NAVIER-STOKES EQUATIONS*
}

\author{
Huaijun Yang and Dongyang Shi ${ }^{1)}$ \\ School of Mathematics, Zhengzhou University of Aeronautics, Zhengzhou 450046, China \\ School of Mathematics and Statistics, Zhengzhou University, Zhengzhou 450001, China \\ Email: huaijunyang@zua.edu.cn, shi_dy@zzu.edu.cn
}

\begin{abstract}
In this paper, the unconditional error estimates are presented for the time-dependent Navier-Stokes equations by the bilinear-constant scheme. The corresponding optimal error estimates for the velocity and the pressure are derived unconditionally, while the previous works require certain time-step restrictions. The analysis is based on an iterated timediscrete system, with which the error function is split into a temporal error and a spatial error. The $\tau$-independent ( $\tau$ is the time stepsize) error estimate between the numerical solution and the solution of the time-discrete system is proven by a rigorous analysis, which implies that the numerical solution in $L^{\infty}$-norm is bounded. Thus optimal error estimates can be obtained in a traditional way. Numerical results are provided to confirm the theoretical analysis.
\end{abstract}

Mathematics subject classification: 65M60, 65M12, 65N15.

Key words: Navier-Stokes equations, Unconditionally optimal error estimates, Bilinearconstant scheme, Time-discrete system.

\section{Introduction}

In this paper, we pay our attention to the following time-dependent incompressible NavierStokes equation in two dimensions:

$$
\begin{aligned}
& \boldsymbol{u}_{t}-\nu \Delta \boldsymbol{u}+(\boldsymbol{u} \cdot \nabla) \boldsymbol{u}+\nabla p=\boldsymbol{f}, \quad(\boldsymbol{x}, t) \in \Omega \times(0, T] \\
& \nabla \cdot \boldsymbol{u}=0, \quad(\boldsymbol{x}, t) \in \Omega \times(0, T], \\
& \boldsymbol{u}(\boldsymbol{x}, t)=0, \quad(\boldsymbol{x}, t) \in \partial \Omega \times(0, T], \\
& \boldsymbol{u}(\boldsymbol{x}, 0)=\boldsymbol{u}_{0}(\boldsymbol{x}), \quad \boldsymbol{x} \in \Omega,
\end{aligned}
$$

where $\Omega \subset \mathbb{R}^{2}$ is a rectangular domain with boundary $\partial \Omega$ and $\boldsymbol{x}=\left(x_{1}, x_{2}\right) . \quad \boldsymbol{u}=\left(u_{1}, u_{2}\right)$ represents the velocity vector, $p$ the pressure, $\boldsymbol{f}=\left(f_{1}, f_{2}\right)$ the body force, $\nu=1 / R e$ the viscosity coefficient and $R e$ is the Reynolds number.

It is well known that the time-dependent incompressible Navier-Stokes equations is a very important system in the mathematical physics and the fluid mechanics fields. In the past several decades, a lot of efforts have been devoted to the development of efficient numerical approximations for solving this system [1-9]. In particular, a new fully-discrete finite element

\footnotetext{
${ }^{*}$ Received June 19, 2020 / Accepted July 23, 2020 /

Published online February 27, 2021 /

1) Corresponding author
} 
nonlinear Galerkin method was studied to the long time integration of the Navier-Stokes equations in [5] through the spatial discretization based on two-grid finite element technique and the time discretization based on Euler explicit scheme with variable time stepsize. However, a certain time step constraint was required so as to obtain the boundedness and convergence of the above method. In [6], the Lagrange-Galerkin mixed finite element approximation of the Navier-Stokes equations was discussed and the corresponding optimal estimate was derived with the time stepsize restriction $\tau=\mathcal{O}\left(h^{\sigma}\right)$, where $\sigma>(n-1) / 2$ and $n$ denotes the dimensions of the domain $\Omega$. A suboptimal convergence rate $\mathcal{O}\left(h+\tau+h^{2} / \tau\right)$ was derived in [8] by a characteristics type Galerkin finite element methods. The restriction could become more serious when the problem was considered in a high-dimensional space and/or with a non-uniform mesh.

On the other hand, for the nonlinear problems, linearized (semi)-implicit schemes are more efficient since at each time step, the schemes only require solving the linear systems. However, the time step restriction condition of the linearized schemes arising from the error analysis is always a crucial issue (see [10]- [14]). In addition, the $L^{\infty}$ boundness of the numerical solution is an essential condition in the error analysis. Most previous works require certain time step restrictions when the inverse inequality is used to bound the numerical solution. Therefore, there have been some attempts to reduce the time step restriction conditions. For example, a new approach was introduced in [15] and [16] to get unconditional stability and optimal error estimates of a linearized backward Euler Galerkin/Galerkin-mixed finite element methods for the time-dependent Joule heating equations and the incompressible miscible flow in porous media, respectively. This new approach is based on a new error splitting technique by a corresponding time-discrete system. Then, with the proved certain regularity of the solution of the time-discrete system, it follows that

$$
\left\|\boldsymbol{U}_{h}^{n}\right\|_{0, \infty} \leq\left\|R_{h} \boldsymbol{U}^{n}\right\|_{0, \infty}+\left\|R_{h} \boldsymbol{U}^{n}-\boldsymbol{U}_{h}^{n}\right\|_{0, \infty} \leq C+C h^{-d / 2} h^{r+1},
$$

where $\boldsymbol{U}_{h}^{n}$ is the finite element solution, $R_{h}$ is the Galerkin projection, $d$ is the dimensions of $\Omega$ and $r$ is the degree of piecewise polynomial. Thus, the boundedness of the numerical solution $\boldsymbol{U}_{h}$ in $L^{\infty}$-norm can be derived without any time step restriction. Subsequently, this approach has been applied to many other problems [17]- [22] to study the convergence or superconvergence of the numerical schemes and to deduce the error estimates almost unconditionally (i.e., the step sizes $h, \tau \leq s_{0}$ for some small positive constant $s_{0}$ ). Moreover, in [23], the unconditional stability and error estimates of modified characteristics finite element methods were researched for the time-dependent Navier-Stokes equations. However, the boundary $\partial \Omega$ of $\Omega$ should belong to $C^{2}$ due to the boundedness of numerical solution $\boldsymbol{U}_{h}^{n}$ in $W^{1, \infty}$-norm used in the error analysis.

In this paper, the unconditionally optimal error estimates are investigated for the timedependent Navier-Stokes equations with Lipschitz boundary $\partial \Omega$, which is weaker than that in [23]. The spatial discretization is approximated by a low order conforming bilinear-constant mixed finite element method [24,25], and the time discretization is approximated by semiimplicit Euler scheme. The analysis is based on an error splitting technique proposed in $[15,16]$ with a time-discrete system. More precisely, the $\tau$-independent error estimate is first derived for the time-discrete system, then the numerical solution in $L^{\infty}$-norm can be bounded in terms of the mathematical induction and inverse inequality, which lead to the unconditionally optimal error estimates are achieved in a routine way.

The rest of this paper is organized as follows. In section 2, we introduce some notations and preliminaries. Moreover, we also present the linearized semi-implicit Euler Galerkin scheme and the main results. The temporal and the spatial error estimates are established in section 
3 and section 4 , respectively. In section 5 , the proof of the main result is presented. Finally, in section 6 , numerical results are provided to verify the theoretical analysis.

\section{Preliminaries and Main Result}

We will use standard notations for the Sobolev spaces $H^{m}(\Omega), m \geq 0$ (cf. [26]) with the norm $\|\cdot\|_{m}$ and seminorm $|\cdot|_{m}$. In the case $m=0$, then $H^{0}(\Omega)=L^{2}(\Omega)$, the norm and inner product are denoted by $\|\cdot\|_{0}$ and $(\cdot, \cdot)$, respectively. For any Banach space $X$ and $I=[0, T]$, let $L^{p}(I ; X)$ be the space of all measurable function $f: I \rightarrow X$ with the norm

$$
\|f\|_{L^{p}(I ; X)}= \begin{cases}\left(\int_{0}^{T}\|f\|_{X}^{p} d t\right)^{\frac{1}{p}}, & 1 \leq p<\infty \\ \operatorname{esssup}_{t \in I}\|f\|_{X}, & p=\infty\end{cases}
$$
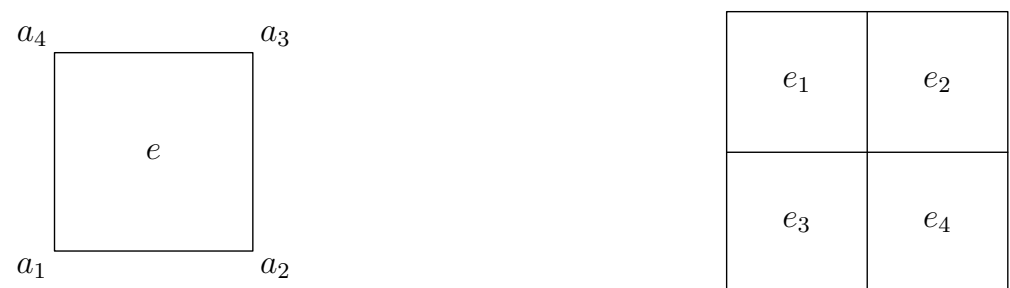

Fig. 2.1. The element $e(l e f t)$ and $\tilde{e}(r i g h t)$.

Let $\mathcal{T}_{h}=\{e\}$ be a uniform rectangular mesh over $\Omega$ with mesh size $h$. For a given element $e \in \mathcal{T}_{h}$, its four nodes are denoted by $a_{i}=\left(x_{1 i}, x_{2 i}\right), i=1,2,3,4$ in the counterclockwise order (see Fig. 2.1). For the velocity, we choose $\boldsymbol{V}_{h}$ as the general bilinear finite element space. For the pressure, we assume that the subdivision $\mathcal{T}_{h}$ is obtained from $\mathcal{T}_{2 h}=\{\tilde{e}\}$ by dividing each element of $\mathcal{T}_{2 h}$ into four small congruent rectangles. Let $P_{h}^{\prime}$ consist of piecewise constant functions with respect to $\mathcal{T}_{h}$ and the local basis functions for $P_{h}^{\prime}$ on a $2 \times 2$-patch of $\tilde{e}$ (see Fig. 2.1) are indicated in Fig. 2.2. Then, the finite element space for pressure is defined by $P_{h}^{\prime} \cap L_{0}^{2}(\Omega)$. In the following discussion we always assume that $\tilde{e}=\cup_{i=1}^{4} e_{i} \in \mathcal{T}_{2 h}$ with $e_{i} \in \mathcal{T}_{h}(1 \leq i \leq 4)$ (see Fig. 2.1). Thus $\boldsymbol{V}_{h}$ and $P_{h}$ for the bilinear-constant scheme are described by (cf. [24])

$$
\begin{aligned}
& \boldsymbol{V}_{h}=\left\{\boldsymbol{v} \in(C(\bar{\Omega}))^{2}:\left.\boldsymbol{v}\right|_{e} \in\left(Q_{1}(e)\right)^{2},\left.\boldsymbol{v}\right|_{\partial \Omega}=0, e \in \mathcal{T}_{h}\right\}, \\
& P_{h}=\left\{p \in L_{0}^{2}(\Omega):\left.p\right|_{\tilde{e}}=\sum_{i=1}^{3} \lambda_{i}^{\tilde{e}} \varphi_{i}^{\tilde{e}}, \sum_{\tilde{e} \in \mathcal{T}_{2 h}} \lambda_{1}^{\tilde{e}}=0, \tilde{e} \in \mathcal{T}_{2 h}\right\},
\end{aligned}
$$

where $Q_{1}$ denotes the space of all polynomials of degree $\leq 1$ with respect to each of the two variables $x_{1}$ and $x_{2}$, and

$$
L_{0}^{2}(\Omega)=\left\{q \in L^{2}(\Omega): \int_{\Omega} q d x_{1} d x_{2}=0\right\} .
$$

Moreover, let $0=t_{0}<t_{1}<\cdots<t_{N}=T$ be a given uniform partition of the time interval with time step $\tau=T / N$ and $t_{n}=n \tau, n=0,1, \cdots, N$. For a smooth function $u$ defined on $[0, T]$, denote

$$
u^{n}=u\left(t_{n}\right), \quad D_{\tau} u^{n}=\frac{u^{n}-u^{n-1}}{\tau} .
$$


Now, we present the following linearized backward Euler fully-discrete scheme: for given $\left(\boldsymbol{U}_{h}^{n-1}, P_{h}^{n-1}\right) \in \boldsymbol{V}_{h} \times P_{h}$, find $\left(\boldsymbol{U}_{h}^{n}, P_{h}^{n}\right) \in \boldsymbol{V}_{h} \times P_{h}$, such that

$$
\begin{aligned}
\left(D_{\tau} \boldsymbol{U}_{h}^{n}, \boldsymbol{v}_{h}\right) & +\nu\left(\nabla \boldsymbol{U}_{h}^{n}, \nabla \boldsymbol{v}_{h}\right)+\left(\left(\boldsymbol{U}_{h}^{n-1} \cdot \nabla\right) \boldsymbol{U}_{h}^{n}, \boldsymbol{v}_{h}\right) \\
& -\left(P_{h}^{n}, \nabla \cdot \boldsymbol{v}_{h}\right)=\left(\boldsymbol{f}^{n}, v_{h}\right), \quad \forall \boldsymbol{v}_{h} \in \boldsymbol{V}_{h}, \\
\left(\nabla \cdot \boldsymbol{U}_{h}^{n}, q_{h}\right) & =0, \quad \forall q_{h} \in P_{h}
\end{aligned}
$$

with the initial approximation $U_{h}^{0}=\Pi_{h} \boldsymbol{u}_{0}(\boldsymbol{x})$, in which $\Pi_{h}$ is the Lagrange interpolation operator on $\boldsymbol{V}_{h}$.

\begin{tabular}{|l|l|}
\hline 1 & 1 \\
\hline 1 & 1 \\
\hline
\end{tabular}

\begin{tabular}{|l|l|}
\hline 1 & -1 \\
\hline 1 & -1 \\
\hline \multicolumn{2}{|c|}{$\varphi_{2}^{\tilde{e}}$} \\
\hline
\end{tabular}

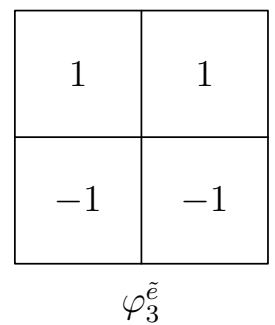

Fig. 2.2. Local basis functions of $P_{h}^{\prime}$.

It has been shown in $[1,24]$ that the bilinear-constant scheme satisfies the discrete BabuškaBrezzi condition, i.e., there exists a constant $\beta>0$, such that

$$
\sup _{0 \neq \boldsymbol{v}_{h} \in \boldsymbol{V}_{h}} \frac{\left(q_{h}, \nabla \cdot \boldsymbol{v}_{h}\right)}{\left\|\boldsymbol{v}_{h}\right\|_{1}} \geq \beta\left\|q_{h}\right\|_{0}, \quad \forall q_{h} \in P_{h} .
$$

Moreover, we introduce the following Galerkin projection $R_{h} \boldsymbol{u} \in \boldsymbol{V}_{h}$ and $Q_{h} p \in P_{h}$ satisfying

$$
\begin{aligned}
& \nu\left(\nabla\left(R_{h} \boldsymbol{u}-\boldsymbol{u}\right), \nabla \boldsymbol{v}_{h}\right)-\left(Q_{h} p-p, \nabla \cdot \boldsymbol{v}_{h}\right)=0, \quad \forall \boldsymbol{v}_{h} \in \boldsymbol{V}_{h}, \\
& \left(\nabla \cdot\left(R_{h} \boldsymbol{u}-\boldsymbol{u}\right), q_{h}\right)=0, \quad \forall q_{h} \in P_{h} .
\end{aligned}
$$

From $[1,27,28]$, we have

$$
\begin{aligned}
& \left\|\boldsymbol{u}-R_{h} \boldsymbol{u}\right\|_{0}+h\left\|\nabla\left(\boldsymbol{u}-R_{h} \boldsymbol{u}\right)\right\|_{0}+h\left\|p-Q_{h} p\right\|_{0} \\
\leq & C h^{2}\left(\|\boldsymbol{u}\|_{2}+\|p\|_{1}\right), \quad \boldsymbol{u} \in\left(H_{0}^{1}(\Omega)\right)^{2} \cap\left(H^{2}(\Omega)\right)^{2}, \quad p \in L_{0}^{2}(\Omega) \cap H^{1}(\Omega)
\end{aligned}
$$

and

$$
\left\|R_{h} \boldsymbol{u}\right\|_{0, \infty} \leq C\left(\|\boldsymbol{u}\|_{2}+\|p\|_{1}\right)
$$

Here, we recall some lemmas, which are necessary to the error estimates.

Lemma 2.1 ( $H^{2}$ regularity of the Stokes equations [1]). Assume that $\Omega$ is a convex polygon in $\mathbb{R}^{2}$ and $\boldsymbol{g} \in\left(L^{2}(\Omega)\right)^{2}$, then the steady Stokes system

$$
\begin{aligned}
& -\nu \Delta \boldsymbol{v}+\nabla q=\boldsymbol{g}, \quad \boldsymbol{x} \in \Omega, \\
& \nabla \cdot \boldsymbol{v}=0, \quad \boldsymbol{x} \in \Omega, \\
& \boldsymbol{v}=0, \quad \boldsymbol{x} \in \partial \Omega,
\end{aligned}
$$

admits a unique solution $(\boldsymbol{v}, q) \in\left(H^{2}(\Omega)\right)^{2} \times H^{1}(\Omega)$ with $\int_{\Omega} q d \boldsymbol{x}=0$ and there exists a constant $C$ independent of $\boldsymbol{g}$ such that

$$
\|\boldsymbol{v}\|_{2}+\|q\|_{1} \leq C\|\boldsymbol{g}\|_{0}
$$


Lemma 2.2 (Discrete Gronwall inequality [4]). Let $\tau, C$ and $\left\{a_{n}\right\},\left\{b_{n}\right\},\left\{c_{n}\right\},\left\{d_{n}\right\}$ be nonnegative numbers such that

$$
a_{n}+\tau \sum_{i=0}^{n} b_{i} \leq \tau \sum_{i=0}^{n} d_{i} a_{i}+\tau \sum_{i=0}^{n} c_{i}+C,
$$

for given $n \geq 0$. Suppose that $\tau d_{i}<1$ for all $i$. Then

$$
a_{n}+\tau \sum_{i=0}^{n} b_{i} \leq \exp \left(\tau \sum_{i=0}^{n} \frac{d_{i}}{1-\tau d_{i}}\right)\left(\tau \sum_{i=0}^{n} c_{i}+C\right) .
$$

In the rest part of this paper, as [22], we assume that the solution $(\boldsymbol{u}, p)$ to the initial boundary value problem (1.1)-(1.4) exists and satisfies

$$
\left\|\boldsymbol{u}_{0}\right\|_{H^{2}}+\|\boldsymbol{u}\|_{L^{\infty}\left(H^{2} \cap W^{1, \infty}\right)}+\left\|\boldsymbol{u}_{t}\right\|_{L^{2}\left(H^{2}\right)}+\|p\|_{L^{\infty}\left(H^{1}\right)}+\left\|p_{t}\right\|_{L^{2}\left(H^{1}\right)} \leq C .
$$

We present our main result on error estimates in the following theorem and prove it later.

Theorem 2.1. Suppose that system (1.1)-(1.4) has a unique solution (u,p) satisfying (2.10). Then, there exist positive constants $\tau_{0}$ and $h_{0}$ such that when $\tau \leq \tau_{0}$ and $h \leq h_{0}$, the finite element system (2.2)-(2.3) admits a unique solution $\left(\boldsymbol{U}_{h}^{n}, P_{h}^{n}\right), n=1,2, \ldots, N$, which satisfies

$$
\begin{aligned}
& \max _{0 \leq n \leq N}\left\|\boldsymbol{u}^{n}-\boldsymbol{U}_{h}^{n}\right\|_{0}^{2} \leq C\left(h^{4}+\tau^{2}\right), \\
& \max _{0 \leq n \leq N}\left\|\nabla\left(\boldsymbol{u}^{n}-\boldsymbol{U}_{h}^{n}\right)\right\|_{0}^{2} \leq C\left(h^{2}+\tau^{2}\right), \\
& \tau \sum_{n=1}^{N}\left\|p^{n}-P_{h}^{n}\right\|_{0}^{2} \leq C\left(h^{2}+\tau^{2}\right),
\end{aligned}
$$

here and later, $C$ is a positive constant independent of $n, h$ and $\tau$.

\section{The Temporal Error Estimates}

To prove the results in Theorem 2.1, we introduce the following time-discrete system (cf. [23])

$$
\begin{aligned}
& D_{\tau} \boldsymbol{U}^{n}-\nu \Delta \boldsymbol{U}^{n}+\left(\boldsymbol{U}^{n-1} \cdot \nabla\right) \boldsymbol{U}^{n}+\nabla P^{n}=\boldsymbol{f}^{n}, \\
& \nabla \cdot \boldsymbol{U}^{n}=0, \\
& \left.\boldsymbol{U}^{n}\right|_{\partial \Omega}=0, \\
& \boldsymbol{U}^{0}=\boldsymbol{u}_{0} .
\end{aligned}
$$

Then, we have the following error estimates in the temporal direction.

Theorem 3.1. Suppose that system (1.1)-(1.4) has a unique solution (u,p) satisfying (2.10). Then the system (3.1)-(3.4) also admits a unique solution $\left(\boldsymbol{U}^{n}, P^{n}\right), n=1,2, \ldots, N$, and there exists a positive constant $\tau^{*}$ such that when $\tau \leq \tau^{*}$

$$
\begin{aligned}
& \max _{0 \leq n \leq N}\left\|\boldsymbol{e}^{n}\right\|_{0}+\left\|\nabla \boldsymbol{e}^{n}\right\|_{1} \leq C \tau, \\
& \max _{0 \leq n \leq N}\left\|\boldsymbol{U}^{n}\right\|_{2}+\max _{0 \leq n \leq N}\left\|P^{n}\right\|_{1} \leq C, \\
& \tau \sum_{n=1}^{N}\left\|D_{\tau} \boldsymbol{U}^{n}\right\|_{2}^{2}+\tau \sum_{n=1}^{N}\left\|D_{\tau} P^{n}\right\|_{1}^{2} \leq C .
\end{aligned}
$$


Proof. Let $\boldsymbol{e}^{n}=\boldsymbol{u}^{n}-\boldsymbol{U}^{n}$, from (1.1)-(1.2), at $t=t_{n}$, we have

$$
\begin{aligned}
& D_{\tau} \boldsymbol{u}^{n}-\nu \Delta \boldsymbol{u}^{n}+\left(\boldsymbol{u}^{n-1} \cdot \nabla\right) \boldsymbol{u}^{n}+\nabla p^{n}=\boldsymbol{f}^{n}+\boldsymbol{R}_{1}^{n}+\boldsymbol{R}_{2}^{n}, \\
& \nabla \cdot \boldsymbol{u}^{n}=0,
\end{aligned}
$$

where

$$
\boldsymbol{R}_{1}^{n}=D_{\tau} \boldsymbol{u}^{n}-\boldsymbol{u}_{t}^{n}, \quad \boldsymbol{R}_{2}^{n}=\left(\left(\boldsymbol{u}^{n-1}-\boldsymbol{u}^{n}\right) \cdot \nabla\right) \boldsymbol{u}^{n}
$$

satisfying

$$
\tau \sum_{n=0}^{N}\left\|\boldsymbol{R}_{1}^{n}\right\|_{0}^{2}+\tau \sum_{n=0}^{N}\left\|\boldsymbol{R}_{2}^{n}\right\|_{0}^{2} \leq C \tau^{2} .
$$

Now, we start to prove the following inequality

$$
\left\|e^{n}\right\|_{2} \leq 1
$$

by mathematical induction for $n=0,1, \ldots, N$.

In fact, since $\boldsymbol{U}^{0}=\boldsymbol{u}^{0}$, the inequality (3.11) holds for $n=0$.

We assume that (3.11) also holds for $n \leq k-1$ for some integer $k>0$. Thus, we have

$$
\begin{aligned}
\left\|\boldsymbol{U}^{n}\right\|_{0, \infty} & \leq\left\|\boldsymbol{u}^{n}\right\|_{0, \infty}+\left\|\boldsymbol{e}^{n}\right\|_{0, \infty} \\
& \leq\left\|\boldsymbol{u}^{n}\right\|_{0, \infty}+C\left\|\boldsymbol{e}^{n}\right\|_{2} \leq C .
\end{aligned}
$$

For $n=k$, from (3.8)-(3.9) and (3.1)-(3.2), we have

$$
\begin{aligned}
& D_{\tau} \boldsymbol{e}^{n}-\nu \Delta \boldsymbol{e}^{n}+\boldsymbol{\Lambda}^{n}+\nabla\left(p^{n}-P^{n}\right)=\boldsymbol{R}_{1}^{n}+\boldsymbol{R}_{2}^{n}, \\
& \nabla \cdot \boldsymbol{e}^{n}=0,
\end{aligned}
$$

where $\boldsymbol{\Lambda}^{n}=\left(\boldsymbol{u}^{n-1} \cdot \nabla\right) \boldsymbol{u}^{n}-\left(\boldsymbol{U}^{n-1} \cdot \nabla\right) \boldsymbol{U}^{n}$.

Multiplying (3.13) by $2 \tau D_{\tau} \boldsymbol{e}^{n}$, integrating it over $\Omega$ and noting the (3.14) give

$$
\begin{aligned}
& 2 \tau\left\|D_{\tau} \boldsymbol{e}^{n}\right\|_{0}^{2}+\nu\left(\left\|\nabla \boldsymbol{e}^{n}\right\|_{0}^{2}-\left\|\nabla \boldsymbol{e}^{n-1}\right\|_{0}^{2}\right) \\
\leq & -\left(\boldsymbol{\Lambda}^{n}, 2 \tau D_{\tau} \boldsymbol{e}^{n}\right)+\left(\boldsymbol{R}_{1}^{n}, 2 \tau D_{\tau} \boldsymbol{e}^{n}\right)+\left(\boldsymbol{R}_{2}, 2 \tau D_{\tau} \boldsymbol{e}^{n}\right) .
\end{aligned}
$$

Firstly, we have

$$
\begin{aligned}
\boldsymbol{\Lambda}^{n} & =\left(\boldsymbol{u}^{n-1} \cdot \nabla\right) \boldsymbol{u}^{n}-\left(\boldsymbol{U}^{n-1} \cdot \nabla\right) \boldsymbol{U}^{n} \\
& =\left(\boldsymbol{e}^{n-1} \cdot \nabla\right) \boldsymbol{u}^{n}+\left(\boldsymbol{U}^{n-1} \cdot \nabla\right) \boldsymbol{e}^{n} .
\end{aligned}
$$

Therefore, it follows that

$$
\begin{aligned}
\left(\left(\boldsymbol{e}^{n-1} \cdot \nabla\right) \boldsymbol{u}^{n}, 2 \tau D_{\tau} \boldsymbol{e}^{n}\right) & \leq C \tau\left\|\boldsymbol{e}^{n-1}\right\|_{0,4}\left\|\nabla \boldsymbol{u}^{n}\right\|_{0,4}\left\|D_{\tau} \boldsymbol{e}^{n}\right\|_{0} \\
& \leq C \tau\left\|\nabla \boldsymbol{e}^{n-1}\right\|_{0}\left\|D_{\tau} \boldsymbol{e}^{n}\right\|_{0} \\
& \leq C \tau\left\|\nabla \boldsymbol{e}^{n-1}\right\|_{0}^{2}+\epsilon \tau\left\|D_{\tau} \boldsymbol{e}^{n}\right\|_{0}^{2}
\end{aligned}
$$

and

$$
\begin{aligned}
\left(\left(\boldsymbol{U}^{n-1} \cdot \nabla\right) \boldsymbol{e}^{n}, 2 \tau D_{\tau} \boldsymbol{e}^{n}\right) & \leq C \tau\left\|\boldsymbol{U}^{n-1}\right\|_{0, \infty}\left\|\nabla \boldsymbol{e}^{n}\right\|_{0}\left\|D_{\tau} \boldsymbol{e}^{n}\right\|_{0} \\
& \leq C \tau\left\|\nabla \boldsymbol{e}^{n}\right\|_{0}^{2}+\epsilon \tau\left\|D_{\tau} \boldsymbol{e}^{n}\right\|_{0}^{2}
\end{aligned}
$$


Thus, we obtain

$$
\left|\left(\boldsymbol{\Lambda}^{n}, 2 \tau D_{\tau} \boldsymbol{e}^{n}\right)\right| \leq C \tau\left\|\nabla \boldsymbol{e}^{n}\right\|_{0}^{2}+C \tau\left\|\nabla \boldsymbol{e}^{n-1}\right\|_{0}^{2}+2 \epsilon \tau\left\|D_{\tau} \boldsymbol{e}^{n}\right\|_{0}^{2} .
$$

On the other hand, we have

$$
\begin{aligned}
\left|\left(\boldsymbol{R}_{1}^{n}, 2 \tau D_{\tau} \boldsymbol{e}^{n}\right)\right|+\left|\left(\boldsymbol{R}_{2}^{n}, 2 \tau D_{\tau} \boldsymbol{e}^{n}\right)\right| & \leq C \tau\left(\left\|\boldsymbol{R}_{1}^{n}\right\|_{0}+\left\|\boldsymbol{R}_{2}^{n}\right\|_{0}\right)\left\|D_{\tau} \boldsymbol{e}^{n}\right\|_{0} \\
& \leq C \tau\left(\left\|\boldsymbol{R}_{1}^{n}\right\|_{0}^{2}+\left\|\boldsymbol{R}_{2}^{n}\right\|_{0}^{2}\right)+\epsilon \tau\left\|D_{\tau} \boldsymbol{e}^{n}\right\|_{0}^{2} .
\end{aligned}
$$

Based on the above achievements, it follows that

$$
\begin{aligned}
\left\|\nabla \boldsymbol{e}^{n}\right\|_{0}^{2}-\left\|\nabla \boldsymbol{e}^{n-1}\right\|_{0}^{2}+\tau\left\|D_{\tau} \boldsymbol{e}^{n}\right\|_{0}^{2} & \leq C \tau\left(\left\|\nabla \boldsymbol{e}^{n}\right\|_{0}^{2}+\left\|\nabla \boldsymbol{e}^{n-1}\right\|_{0}^{2}\right) \\
& +C \tau\left(\left\|\boldsymbol{R}_{1}^{n}\right\|_{0}^{2}+\left\|\boldsymbol{R}_{2}^{n}\right\|_{0}^{2}\right),
\end{aligned}
$$

which in turn produces

$$
\left\|\nabla \boldsymbol{e}^{n}\right\|_{0}^{2}+\tau \sum_{\ell=1}^{n}\left\|D_{\tau} \boldsymbol{e}^{\ell}\right\|_{0}^{2} \leq C \tau \sum_{\ell=0}^{n}\left\|\nabla \boldsymbol{e}^{\ell}\right\|_{0}^{2}+C \tau^{2}
$$

where we have used $\boldsymbol{e}^{0}=0$ and (3.10).

Then, according to Lemma 2.2, there exists a small $\tau_{1}$, when $\tau \leq \tau_{1}$ such that

$$
\left\|\nabla \boldsymbol{e}^{n}\right\|_{0}^{2}+\tau \sum_{\ell=1}^{n}\left\|D_{\tau} \boldsymbol{e}^{\ell}\right\|_{0}^{2} \leq C \tau^{2} .
$$

By Pioncaré inequality, we have

$$
\left\|e^{n}\right\|_{0}^{2} \leq C \tau^{2}
$$

Secondly, applying Lemma 2.1 to equations (3.13) and (3.14), we arrive at

$$
\begin{aligned}
& \left\|\boldsymbol{e}^{n}\right\|_{2}+\left\|p^{n}-P^{n}\right\|_{1} \\
\leq & C\left\|D_{\tau} \boldsymbol{e}^{n}\right\|_{0}+C\left\|\left(\boldsymbol{u}^{n-1} \cdot \nabla\right) \boldsymbol{u}^{n}-\left(\boldsymbol{U}^{n-1} \cdot \nabla\right) \boldsymbol{U}^{n}\right\|_{0}+C\left\|\boldsymbol{R}_{1}^{n}\right\|_{0}+C\left\|\boldsymbol{R}_{2}^{n}\right\|_{0} \\
\leq & C\left\|D_{\tau} \boldsymbol{e}^{n}\right\|_{0}+C\left\|\left(\boldsymbol{e}^{n-1} \cdot \nabla\right) \boldsymbol{u}^{n}\right\|_{0}+\left\|\left(\boldsymbol{U}^{n-1} \cdot \nabla\right) \boldsymbol{e}^{n}\right\|_{0}+C\left\|\boldsymbol{R}_{1}^{n}\right\|_{0}+C\left\|\boldsymbol{R}_{2}^{n}\right\|_{0} \\
\leq & C\left\|D_{\tau} \boldsymbol{e}^{n}\right\|_{0}+C\left\|\boldsymbol{e}^{n-1}\right\|_{0,4}\left\|\nabla \boldsymbol{u}^{n}\right\|_{0,4}+C\left\|\boldsymbol{U}^{n-1}\right\|_{0, \infty}\left\|\nabla \boldsymbol{e}^{n}\right\|_{0}+C\left\|\boldsymbol{R}_{1}^{n}\right\|_{0}+C\left\|\boldsymbol{R}_{2}^{n}\right\|_{0} \\
\leq & C\left\|D_{\tau} \boldsymbol{e}^{n}\right\|_{0}+C\left\|\nabla \boldsymbol{e}^{n-1}\right\|_{0}+C\left\|\nabla \boldsymbol{e}^{n}\right\|_{0}+C\left\|\boldsymbol{R}_{1}^{n}\right\|_{0}+C\left\|\boldsymbol{R}_{2}^{n}\right\|_{0} \\
\leq & C\left\|D_{\tau} \boldsymbol{e}^{n}\right\|_{0}+C\left\|\boldsymbol{R}_{1}^{n}\right\|_{0}+C\left\|\boldsymbol{R}_{2}^{n}\right\|_{0}+C \tau,
\end{aligned}
$$

which together with (3.20) and (3.10) implies

$$
\tau \sum_{\ell=1}^{n}\left\|\boldsymbol{e}^{\ell}\right\|_{2}^{2}+\tau \sum_{\ell=1}^{n}\left\|p^{n}-P^{n}\right\|_{1}^{2} \leq C \tau^{2} .
$$

The above inequality also implies that, for $n=k$, there exists a small $\tau_{2}$, when $\tau \leq \tau_{2}$, such that

$$
\left\|e^{n}\right\|_{2} \leq C \tau^{\frac{1}{2}} \leq 1 .
$$

Thus, the mathematical induction (3.11) holds uniformly for $n=0,1, \ldots, N$. 
Finally, from (3.22) and (3.23), we can see that

$$
\left\|\boldsymbol{U}^{n}\right\|_{2} \leq\left\|\boldsymbol{u}^{n}\right\|_{2}+\left\|\boldsymbol{e}^{n}\right\|_{2} \leq C
$$

and

$$
\left\|P^{n}\right\|_{1} \leq\left\|p^{n}\right\|_{1}+\left\|p^{n}-P^{n}\right\|_{1} \leq C .
$$

Moreover, we derive from (3.20) and (3.22) that

$$
\begin{aligned}
\tau \sum_{\ell=1}^{n}\left\|D_{\tau} \boldsymbol{U}^{\ell}\right\|_{2}^{2} & \leq C \tau \sum_{\ell=1}^{n}\left(\left\|D_{\tau} \boldsymbol{u}^{\ell}\right\|_{2}^{2}+\left\|D_{\tau} \boldsymbol{e}^{\ell}\right\|_{2}^{2}\right) \\
& \leq C+C \tau^{-2} \cdot \tau \sum_{\ell=1}^{n}\left\|\boldsymbol{e}^{\ell}\right\|_{2}^{2} \leq C
\end{aligned}
$$

and

$$
\begin{aligned}
\tau \sum_{\ell=0}^{n}\left\|D_{\tau} P^{\ell}\right\|_{1}^{2} & \leq C \tau\left(\sum_{\ell=0}^{n}\left\|D_{\tau} p^{\ell}\right\|_{1}^{2}+\left\|D_{\tau}\left(p^{\ell}-P^{\ell}\right)\right\|_{1}^{2}\right) \\
& \leq C+C \tau^{-2} \cdot \tau \sum_{\ell=1}^{n}\left\|p^{\ell}-P^{\ell}\right\|_{1}^{2} \leq C .
\end{aligned}
$$

Thus the desired results are obtained by choosing $\tau^{*} \leq \min \left\{\tau_{1}, \tau_{2}\right\}$. The proof is complete.

\section{The Spatial Error Estimates}

In this section, we investigative the error estimates in spatial direction.

The weak formulation of (3.1)-(3.4) reads as:

$$
\begin{aligned}
\left(D_{\tau} \boldsymbol{U}^{n}, \boldsymbol{v}\right) & +\nu\left(\nabla \boldsymbol{U}^{n}, \nabla \boldsymbol{v}\right)+\left(\left(\boldsymbol{U}^{n-1} \cdot \nabla\right) \boldsymbol{U}^{n}, \boldsymbol{v}\right) \\
& -\left(P^{n}, \nabla \cdot \boldsymbol{v}\right)=\left(\boldsymbol{f}^{n}, \boldsymbol{v}\right), \quad \forall \boldsymbol{v} \in\left(H_{0}^{1}(\Omega)\right)^{2}, \\
\left(\nabla \cdot \boldsymbol{U}^{n}, q\right) & =0, \quad \forall q \in L_{0}^{2}(\Omega) .
\end{aligned}
$$

Theorem 4.1. Suppose that system (1.1)-(1.4) has a unique solution (u,p) satisfying (2.10). Then, there exist positive constants $\tau^{* *}$ and $h_{1}$ such that when $\tau \leq \tau^{* *}$ and $h \leq h_{1}$, the finite element system (2.2)-(2.3) admits a unique solution $\left(\boldsymbol{U}_{h}^{n}, P_{h}^{n}\right), n=1,2, \ldots, N$, which satisfies

$$
\begin{aligned}
& \max _{0 \leq n \leq N}\left\|R_{h} \boldsymbol{U}^{n}-\boldsymbol{U}_{h}^{n}\right\|_{0} \leq C h^{2}, \\
& \max _{0 \leq n \leq N}\left\|\boldsymbol{U}_{h}^{n}\right\|_{0, \infty} \leq C .
\end{aligned}
$$

Proof. For each given $n$, it is easy to see that (2.2)-(2.3) is a finite element system of a Stokes type problem, thus the system has a unique solution $\left(\boldsymbol{U}_{h}^{n}, P_{h}^{n}\right) \in \boldsymbol{V}_{h} \times P_{h}$ for $n=1,2, \ldots, N$.

In terms of (2.5) and (2.6), we split the error between $U^{n}$ and $U_{h}^{n}$ as:

$$
\boldsymbol{U}^{n}-\boldsymbol{U}_{h}^{n}=\boldsymbol{U}^{n}-R_{h} \boldsymbol{U}^{n}+R_{h} \boldsymbol{U}^{n}-\boldsymbol{U}_{h}^{n}:=\boldsymbol{U}^{n}-R_{h} \boldsymbol{U}^{n}+\boldsymbol{\theta}^{n} .
$$

We first prove the following error estimate

$$
\left\|\boldsymbol{\theta}^{n}\right\|_{0} \leq C_{0} h^{2}, \quad n=0,1, \ldots, N
$$


by mathematical induction.

In fact, for $n=0$, note that $\boldsymbol{U}_{h}^{0}=\Pi_{h} \boldsymbol{u}_{0}$, it is easy to see that (4.5) holds at the initial time step.

Now, we assume that (4.5) holds for $n \leq k-1$ for some integer $k>0$, then we have

$$
\left\|\boldsymbol{U}_{h}^{n}\right\|_{0, \infty} \leq\left\|\boldsymbol{\theta}^{n}\right\|_{0, \infty}+\left\|R_{h} \boldsymbol{U}^{n}\right\|_{0, \infty} \leq C h^{-1}\left\|\boldsymbol{\theta}^{n}\right\|_{0}+C\left(\left\|\boldsymbol{U}^{n}\right\|_{2}+\left\|P^{n}\right\|_{1}\right) \leq C,
$$

when $h \leq h_{1}$, such that $C C_{0} h_{1} \leq 1$.

Moreover, from (4.1)-(4.2) and (2.2)-(2.3), we have

$$
\begin{aligned}
& \left(D_{\tau} \boldsymbol{\theta}^{n}, \boldsymbol{v}_{h}\right)+\nu\left(\nabla \boldsymbol{\theta}^{n}, \nabla \boldsymbol{v}_{h}\right)-\left(Q_{h} P^{n}-P_{h}^{n}, \nabla \cdot \boldsymbol{v}_{h}\right) \\
& \quad=-\left(D_{\tau}\left(\boldsymbol{U}^{n}-R_{h} \boldsymbol{U}^{n}\right), \boldsymbol{v}_{h}\right)-\left(\left(\boldsymbol{U}^{n-1} \cdot \nabla\right) \boldsymbol{U}^{n}-\left(\boldsymbol{U}_{h}^{n-1} \cdot \nabla\right) \boldsymbol{U}_{h}^{n}, \boldsymbol{v}_{h}\right), \quad \forall \boldsymbol{v}_{h} \in \boldsymbol{V}_{h}, \\
& \left(\nabla \cdot \boldsymbol{\theta}^{n}, q_{h}\right)=0, \quad \forall q_{h} \in P_{h} .
\end{aligned}
$$

By taking $\boldsymbol{v}_{h}=2 \tau \boldsymbol{\theta}^{n}$ in (4.7) and $q_{h}=Q_{h} P^{n}-P_{h}^{n}$ in (4.8), we derive

$$
\begin{aligned}
& \left\|\boldsymbol{\theta}^{n}\right\|_{0}^{2}-\left\|\boldsymbol{\theta}^{n-1}\right\|_{0}^{2}+2 \tau \nu\left\|\nabla \boldsymbol{\theta}^{n}\right\|_{0}^{2} \\
\leq & -\left(D_{\tau}\left(\boldsymbol{U}^{n}-R_{h} \boldsymbol{U}^{n}\right), 2 \tau \boldsymbol{\theta}^{n}\right)-\left(\left(\boldsymbol{U}^{n-1} \cdot \nabla\right) \boldsymbol{U}^{n}-\left(\boldsymbol{U}_{h}^{n-1} \cdot \nabla\right) \boldsymbol{U}_{h}^{n}, 2 \tau \boldsymbol{\theta}^{n}\right) .
\end{aligned}
$$

Firstly, from (2.7), we can obtain

$$
\begin{aligned}
\left|\left(D_{\tau}\left(\boldsymbol{U}^{n}-R_{h} \boldsymbol{U}^{n}\right), 2 \tau \boldsymbol{\theta}^{n}\right)\right| & \leq C \tau\left\|D_{\tau}\left(\boldsymbol{U}^{n}-R_{h} \boldsymbol{U}^{n}\right)\right\|_{0}\left\|\boldsymbol{\theta}^{n}\right\|_{0} \\
& \leq C \tau h^{2}\left(\left\|D_{\tau} \boldsymbol{U}^{n}\right\|_{2}+\left\|D_{\tau} P^{n}\right\|_{1}\right)\left\|\boldsymbol{\theta}^{n}\right\|_{0} \\
& \leq C \tau h^{4}\left(\left\|D_{\tau} \boldsymbol{U}^{n}\right\|_{2}^{2}+\left\|D_{\tau} P^{n}\right\|_{1}^{2}\right)+\epsilon \tau\left\|\nabla \boldsymbol{\theta}^{n}\right\|_{0} .
\end{aligned}
$$

On the other hand, we have

$$
\begin{aligned}
& \left(\boldsymbol{U}^{n-1} \cdot \nabla\right) \boldsymbol{U}^{n}-\left(\boldsymbol{U}_{h}^{n-1} \cdot \nabla\right) \boldsymbol{U}_{h}^{n} \\
= & \left(\left(\boldsymbol{U}^{n-1}-\boldsymbol{U}_{h}^{n-1}\right) \cdot \nabla\right) \boldsymbol{U}^{n}+\left(\boldsymbol{U}_{h}^{n-1} \cdot \nabla\right)\left(\boldsymbol{U}^{n}-\boldsymbol{U}_{h}^{n}\right) \\
= & \left(\left(\boldsymbol{U}^{n-1}-R_{h} \boldsymbol{U}^{n-1}\right) \cdot \nabla\right) \boldsymbol{U}^{n}+\left(\left(R_{h} \boldsymbol{U}^{n-1}-\boldsymbol{U}_{h}^{n-1}\right) \cdot \nabla\right) \boldsymbol{U}^{n} \\
& +\left(\boldsymbol{U}_{h}^{n-1} \cdot \nabla\right)\left(\boldsymbol{U}^{n}-R_{h} \boldsymbol{U}^{n}\right)+\left(\boldsymbol{U}_{h}^{n-1} \cdot \nabla\right)\left(R_{h} \boldsymbol{U}^{n}-\boldsymbol{U}_{h}^{n}\right) \\
: & \sum_{k=1}^{4} \boldsymbol{A}_{k} .
\end{aligned}
$$

For $\boldsymbol{A}_{1}$, note that

$$
\left\|\boldsymbol{U}-R_{h} \boldsymbol{U}\right\|_{0} \leq C h^{2}\left(\|\boldsymbol{U}\|_{2}+\|P\|_{1}\right) \leq C h^{2},
$$

we have

$$
\begin{aligned}
\left(\boldsymbol{A}_{1}, 2 \tau \boldsymbol{\theta}^{n}\right) & \leq C \tau\left\|\boldsymbol{U}^{n-1}-R_{h} \boldsymbol{U}^{n-1}\right\|_{0}\left\|\nabla \boldsymbol{U}^{n}\right\|_{0,4}\left\|\boldsymbol{\theta}^{n}\right\|_{0,4} \\
& \leq C \tau h^{2}\left\|\nabla \boldsymbol{\theta}^{n}\right\|_{0} \leq C \tau h^{4}+\epsilon \tau\left\|\nabla \boldsymbol{\theta}^{n}\right\|_{0}^{2},
\end{aligned}
$$

and

$$
\begin{aligned}
\left(\boldsymbol{A}_{2}, 2 \tau \boldsymbol{\theta}^{n}\right) & \leq C \tau\left\|R_{h} \boldsymbol{U}^{n-1}-\boldsymbol{U}_{h}^{n-1}\right\|_{0}\left\|\nabla \boldsymbol{U}^{n}\right\|_{0,4}\left\|\boldsymbol{\theta}^{n}\right\|_{0,4} \\
& \leq C \tau\left\|\boldsymbol{\theta}^{n-1}\right\|_{0}\left\|\nabla \boldsymbol{\theta}^{n}\right\|_{0} \leq C \tau\left\|\boldsymbol{\theta}^{n-1}\right\|_{0}^{2}+\epsilon \tau\left\|\nabla \boldsymbol{\theta}^{n}\right\|_{0}^{2} .
\end{aligned}
$$


For $\boldsymbol{A}_{3}$, we rewrite it as

$$
\begin{aligned}
\boldsymbol{A}_{3}= & \left(\boldsymbol{U}_{h}^{n-1} \cdot \nabla\right)\left(\boldsymbol{U}^{n}-R_{h} \boldsymbol{U}^{n}\right) \\
= & \left(\left(\boldsymbol{U}_{h}^{n-1}-\boldsymbol{U}^{n-1}\right) \cdot \nabla\right)\left(\boldsymbol{U}^{n}-R_{h} \boldsymbol{U}^{n}\right)+\left(\boldsymbol{U}^{n-1} \cdot \nabla\right)\left(\boldsymbol{U}^{n}-R_{h} \boldsymbol{U}^{n}\right) \\
= & \left(\left(\boldsymbol{U}_{h}^{n-1}-R_{h} \boldsymbol{U}^{n-1}\right) \cdot \nabla\right)\left(\boldsymbol{U}^{n}-R_{h} \boldsymbol{U}^{n}\right) \\
& +\left(\left(R_{h} \boldsymbol{U}^{n-1}-\boldsymbol{U}^{n-1}\right) \cdot \nabla\right)\left(\boldsymbol{U}^{n}-R_{h} \boldsymbol{U}^{n}\right) \\
& +\left(\boldsymbol{U}^{n-1} \cdot \nabla\right)\left(\boldsymbol{U}^{n}-R_{h} \boldsymbol{U}^{n}\right):=\sum_{k=1}^{3} \boldsymbol{A}_{3 k} .
\end{aligned}
$$

Then, we have

$$
\begin{aligned}
\left(\boldsymbol{A}_{31}, 2 \tau \boldsymbol{\theta}^{n}\right) & \leq C \tau\left\|\boldsymbol{U}_{h}^{n-1}-R_{h} \boldsymbol{U}^{n-1}\right\|_{0,4}\left\|\nabla\left(\boldsymbol{U}^{n}-R_{h} \boldsymbol{U}^{n}\right)\right\|_{0}\left\|\boldsymbol{\theta}^{n}\right\|_{0,4} \\
& \leq C \tau h^{-\frac{1}{2}}\left\|\boldsymbol{\theta}^{n-1}\right\|_{0}\left(h\left(\left\|\boldsymbol{U}^{n}\right\|_{2}+\left\|P^{n}\right\|_{1}\right)\right) h^{-\frac{1}{2}}\left\|\boldsymbol{\theta}^{n}\right\|_{0} \\
& \leq C \tau\left\|\boldsymbol{\theta}^{n-1}\right\|_{0}\left\|\nabla \boldsymbol{\theta}^{n}\right\|_{0} \leq C \tau\left\|\boldsymbol{\theta}^{n-1}\right\|_{0}^{2}+\epsilon \tau\left\|\nabla \boldsymbol{\theta}^{n}\right\|_{0}^{2}, \\
\left(\boldsymbol{A}_{32}, 2 \tau \boldsymbol{\theta}^{n}\right) & \leq C \tau\left\|R_{h} \boldsymbol{U}^{n-1}-\boldsymbol{U}^{n-1}\right\|_{0}\left\|\nabla\left(\boldsymbol{U}^{n}-R_{h} \boldsymbol{U}^{n}\right)\right\|_{0}\left\|\boldsymbol{\theta}^{n}\right\|_{0, \infty} \\
& \leq C \tau\left(h^{2}\left(\left\|\boldsymbol{U}^{n-1}\right\|_{2}+\left\|P^{n-1}\right\|_{1}\right)\right)\left(h\left(\left\|\boldsymbol{U}^{n}\right\|_{2}+\left\|P^{n}\right\|_{1}\right)\right)\left(h^{-1}\left\|\boldsymbol{\theta}^{n}\right\|_{0}\right) \\
& \leq C \tau h^{2}\left\|\nabla \boldsymbol{\theta}^{n}\right\|_{0} \leq C \tau h^{4}+\epsilon \tau\left\|\nabla \boldsymbol{\theta}^{n}\right\|_{0}^{2} .
\end{aligned}
$$

In addition, by applying Green's formula

$$
\int_{\Omega}(\boldsymbol{u} \cdot \nabla) \boldsymbol{w} \cdot \boldsymbol{v} d \boldsymbol{x}=-\int_{\Omega}(\nabla \cdot \boldsymbol{u}) \boldsymbol{w} \cdot \boldsymbol{v} d \boldsymbol{x}-\int_{\Omega}(\boldsymbol{u} \cdot \nabla) \boldsymbol{v} \cdot \boldsymbol{w} d \boldsymbol{x}+\int_{\partial \Omega}(\boldsymbol{u} \cdot \boldsymbol{n})(\boldsymbol{w} \cdot \boldsymbol{v}) d s
$$

we have

$$
\begin{aligned}
\left(\boldsymbol{A}_{33}, 2 \tau \boldsymbol{\theta}^{n}\right) & =-2 \tau\left(\left(\boldsymbol{U}^{n-1} \cdot \nabla\right) \boldsymbol{\theta}^{n}, \boldsymbol{U}^{n}-\boldsymbol{R}_{h} \boldsymbol{U}^{n}\right) \\
& \leq C \tau\left\|\boldsymbol{U}^{n-1}\right\|_{0, \infty}\left\|\nabla \boldsymbol{\theta}^{n}\right\|_{0}\left\|\boldsymbol{U}^{n}-\boldsymbol{R}_{h} \boldsymbol{U}^{n}\right\|_{0} \\
& \leq C \tau h^{2}\left\|\nabla \boldsymbol{\theta}^{n}\right\|_{0} \leq C \tau h^{4}+\epsilon \tau\left\|\nabla \boldsymbol{\theta}^{n}\right\|_{0}^{2}
\end{aligned}
$$

Hence

$$
\left(\boldsymbol{A}_{3}, 2 \tau \boldsymbol{\theta}^{n}\right) \leq C \tau\left\|\boldsymbol{\theta}^{n-1}\right\|_{0}^{2}+C \tau h^{4}+\epsilon \tau\left\|\nabla \boldsymbol{\theta}^{n}\right\|_{0}^{2} .
$$

For $\boldsymbol{A}_{4}$, by (4.6), we have

$$
\left(\boldsymbol{A}_{4}, 2 \tau \boldsymbol{\theta}^{n}\right) \leq C \tau\left\|\boldsymbol{U}_{h}^{n-1}\right\|_{0, \infty}\left\|\nabla \boldsymbol{\theta}^{n}\right\|_{0}\left\|\boldsymbol{\theta}^{n}\right\|_{0} \leq C \tau\left\|\boldsymbol{\theta}^{n}\right\|_{0}^{2}+\epsilon \tau\left\|\boldsymbol{\theta}^{n}\right\|_{0}^{2} .
$$

Based on the above estimates, we have

$$
\begin{aligned}
& \left\|\boldsymbol{\theta}^{n}\right\|_{0}^{2}-\left\|\boldsymbol{\theta}^{n-1}\right\|_{0}^{2}+2 \tau \nu\left\|\nabla \boldsymbol{\theta}^{n}\right\|_{0}^{2} \\
\leq & C \tau h^{4}+C \tau h^{4}\left(\left\|D_{\tau} \boldsymbol{U}^{n}\right\|_{2}^{2}+\left\|D_{\tau} P^{n}\right\|_{1}^{2}\right)+C \tau\left(\left\|\boldsymbol{\theta}^{n}\right\|_{0}^{2}+\left\|\boldsymbol{\theta}^{n-1}\right\|_{0}^{2}\right)+\epsilon \tau\left\|\nabla \boldsymbol{\theta}^{n}\right\|_{0}^{2} .
\end{aligned}
$$

Then, summing the above inequality up leads to

$$
\begin{aligned}
\left\|\boldsymbol{\theta}^{n}\right\|_{0}^{2}+\tau \nu \sum_{\ell=1}^{n}\left\|\nabla \boldsymbol{\theta}^{\ell}\right\|_{0}^{2} & \leq C \tau \sum_{\ell=0}^{n}\left\|\boldsymbol{\theta}^{\ell}\right\|_{0}^{2}+C \tau h^{4} \sum_{\ell=1}^{n}\left(\left\|D_{\tau} \boldsymbol{U}^{\ell}\right\|_{2}^{2}+\left\|D_{\tau} P^{\ell}\right\|_{1}^{2}\right)+C h^{4} \\
& \leq C \tau \sum_{\ell=0}^{n}\left\|\boldsymbol{\theta}^{\ell}\right\|_{0}^{2}+C h^{4}
\end{aligned}
$$


where we have used $\left\|\boldsymbol{\theta}^{0}\right\|_{0}=\left\|R_{h} \boldsymbol{u}_{0}-\boldsymbol{U}_{h}^{0}\right\|_{0} \leq\left\|R_{h} \boldsymbol{u}_{0}-\boldsymbol{u}_{0}\right\|_{0}+\left\|\boldsymbol{u}_{0}-\Pi_{h} \boldsymbol{u}_{0}\right\|_{0} \leq C h^{2}$. Thanks to Lemma 2.2 , there exists a small $\tau_{3}$, such that

$$
\left\|\boldsymbol{\theta}^{n}\right\|_{0}^{2}+\tau \nu \sum_{\ell=1}^{n}\left\|\nabla \boldsymbol{\theta}^{\ell}\right\|_{0}^{2} \leq C^{*} h^{4}
$$

Taking $C_{0} \geq \sqrt{C^{*}}, \tau^{* *} \leq \min \left\{\tau^{*}, \tau_{3}\right\}, h \leq h_{1}$, we can see that for $n=k$

$$
\left\|\boldsymbol{\theta}^{n}\right\|_{0} \leq C_{0} h^{2} .
$$

Thus, the induction (4.5) holds uniformly for $n=0,1, \ldots, N$, which is the desired result (4.3). Also, we can derive

$$
\begin{aligned}
\left\|\boldsymbol{U}_{h}^{n}\right\|_{0, \infty} & \leq\left\|\boldsymbol{\theta}^{n}\right\|_{0, \infty}+\left\|R_{h} \boldsymbol{U}^{n}\right\|_{0, \infty} \\
& \leq C h^{-1}\left\|\boldsymbol{\theta}^{n}\right\|_{0}+C\left(\left\|\boldsymbol{U}^{n}\right\|_{2}+\left\|P^{n}\right\|_{1}\right) \leq C .
\end{aligned}
$$

The proof is complete.

Remark 4.1. In the above proof, $\partial \Omega$ is only required to be Lipschitz continuous, which is indeed weaker than that of $C^{2}$ in [23].

\section{The Proof of the Theorem 2.1}

In order to prove Theorem 2.1, we rewrite the weak formulation of (1.1)-(1.4) as:

$$
\begin{aligned}
\left(D_{\tau} \boldsymbol{u}^{n}, \boldsymbol{v}_{h}\right) & +\nu\left(\nabla \boldsymbol{u}^{n}, \nabla \boldsymbol{v}_{h}\right)+\left(\left(\boldsymbol{u}^{n-1} \cdot \nabla\right) \boldsymbol{u}^{n}, \boldsymbol{v}_{h}\right)-\left(p^{n}, \nabla \cdot \boldsymbol{v}_{h}\right) \\
& =\left(\boldsymbol{f}^{n}, \boldsymbol{v}_{h}\right)+\left(\boldsymbol{R}_{1}^{n}, \boldsymbol{v}_{h}\right)+\left(\boldsymbol{R}_{2}^{n}, \boldsymbol{v}_{h}\right), \quad \forall \boldsymbol{v}_{h} \in \boldsymbol{V}_{h}, \\
\left(\nabla \cdot \boldsymbol{u}^{n}, q_{h}\right) & =0, \quad \forall q_{h} \in P_{h} .
\end{aligned}
$$

For clarity, let

$$
\begin{aligned}
& \boldsymbol{u}^{n}-\boldsymbol{U}_{h}^{n}=\boldsymbol{u}^{n}-R_{h} \boldsymbol{u}^{n}+R_{h} \boldsymbol{u}^{n}-\boldsymbol{U}_{h}^{n}=: \boldsymbol{u}^{n}-R_{h} \boldsymbol{u}^{n}+\boldsymbol{\theta}_{h}^{n}, \\
& p^{n}-P_{h}^{n}=p^{n}-Q_{h} p^{n}+Q_{h} p^{n}-P_{h}^{n}=: p^{n}-Q_{h} p^{n}+\eta_{h}^{n} .
\end{aligned}
$$

Thus, from (5.1)-(5.2) and (2.2)-(2.3), we obtain the error equations:

$$
\begin{aligned}
& \left(D_{\tau} \boldsymbol{\theta}_{h}^{n}, \boldsymbol{v}_{h}\right)+\nu\left(\nabla \boldsymbol{\theta}_{h}^{n}, \nabla \boldsymbol{v}_{h}\right)-\left(\eta_{h}^{n}, \nabla \cdot \boldsymbol{v}_{h}\right)=-\left(D_{\tau}\left(\boldsymbol{u}^{n}-R_{h} \boldsymbol{u}^{n}\right), \boldsymbol{v}_{h}\right) \\
& \quad-\left(\left(\boldsymbol{u}^{n-1} \cdot \nabla\right) \boldsymbol{u}^{n}-\left(\boldsymbol{U}_{h}^{n-1} \cdot \nabla\right) \boldsymbol{U}_{h}^{n}, \boldsymbol{v}_{h}\right)+\left(\boldsymbol{R}_{1}^{n}, \boldsymbol{v}_{h}\right)+\left(\boldsymbol{R}_{2}^{n}, \boldsymbol{v}_{h}\right), \\
& \left(\nabla \cdot \boldsymbol{\theta}_{h}^{n}, q_{h}\right)=0 .
\end{aligned}
$$

Firstly, we give the error estimate of $\boldsymbol{\theta}_{h}^{n}$ in $L^{2}$-norm. To do this, by taking $\boldsymbol{v}_{h}=\boldsymbol{\theta}_{h}^{n}$ in (5.3) and $q_{h}=\eta_{h}^{n}$ in (5.4) results in

$$
\begin{aligned}
& \frac{1}{2 \tau}\left(\left\|\boldsymbol{\theta}_{h}^{n}\right\|_{0}^{2}-\left\|\boldsymbol{\theta}_{h}^{n-1}\right\|_{0}^{2}\right)+\nu\left\|\nabla \boldsymbol{\theta}_{h}^{n}\right\|_{0}^{2} \\
\leq & -\left(D_{\tau}\left(\boldsymbol{u}^{n}-R_{h} \boldsymbol{u}^{n}\right), \boldsymbol{\theta}_{h}^{n}\right)-\left(\left(\boldsymbol{u}^{n-1} \cdot \nabla\right) \boldsymbol{u}^{n}-\left(\boldsymbol{U}_{h}^{n-1} \cdot \nabla\right) \boldsymbol{U}_{h}^{n}, \boldsymbol{\theta}_{h}^{n}\right) \\
& +\left(\boldsymbol{R}_{1}^{n}, \boldsymbol{\theta}_{h}^{n}\right)+\left(\boldsymbol{R}_{2}^{n}, \boldsymbol{\theta}_{h}^{n}\right) .
\end{aligned}
$$


Then, by (2.7) and Cauchy-Schwarz inequality, we have

$$
\begin{aligned}
\left|\left(D_{\tau}\left(\boldsymbol{u}^{n}-R_{h} \boldsymbol{u}^{n}\right), \boldsymbol{\theta}_{h}^{n}\right)\right| & \leq\left\|D_{\tau}\left(\boldsymbol{u}^{n}-R_{h} \boldsymbol{u}^{n}\right)\right\|_{0}\left\|\boldsymbol{\theta}_{h}^{n}\right\|_{0} \\
& \leq C h^{2}\left(\left\|D_{\tau} \boldsymbol{u}^{n}\right\|_{2}+\left\|D_{\tau} p^{n}\right\|_{1}\right)\left\|\boldsymbol{\theta}_{h}^{n}\right\|_{0} \\
& \leq C h^{4}\left(\left\|D_{\tau} \boldsymbol{u}^{n}\right\|_{2}^{2}+\left\|D_{\tau} p^{n}\right\|_{1}^{2}\right)+C\left\|\boldsymbol{\theta}_{h}^{n}\right\|_{0}^{2}, \\
\left|\left(\boldsymbol{R}_{1}^{n}, \boldsymbol{\theta}_{h}^{n}\right)\right|+\left|\left(\boldsymbol{R}_{2}^{n}, \boldsymbol{\theta}_{h}^{n}\right)\right| & \leq C\left(\left\|\boldsymbol{R}_{1}^{n}\right\|_{0}+\left\|\boldsymbol{R}_{2}^{n}\right\|_{0}\right)\left\|\boldsymbol{\theta}_{h}^{n}\right\|_{0} \\
& \leq C\left(\left\|\boldsymbol{R}_{1}^{n}\right\|_{0}^{2}+\left\|\boldsymbol{R}_{2}^{n}\right\|_{0}^{2}\right)+C\left\|\boldsymbol{\theta}_{h}^{n}\right\|_{0}^{2} .
\end{aligned}
$$

Moreover, it follows that

$$
\begin{aligned}
& \left(\boldsymbol{u}^{n-1} \cdot \nabla\right) \boldsymbol{u}^{n}-\left(\boldsymbol{U}_{h}^{n-1} \cdot \nabla\right) \boldsymbol{U}_{h}^{n} \\
= & \left(\left(\boldsymbol{u}^{n-1}-\boldsymbol{U}_{h}^{n-1}\right) \cdot \nabla\right) \boldsymbol{u}^{n}+\left(\boldsymbol{U}_{h}^{n-1} \cdot \nabla\right)\left(\boldsymbol{u}^{n}-\boldsymbol{U}_{h}^{n}\right) \\
= & \left(\left(\boldsymbol{u}^{n-1}-R_{h} \boldsymbol{u}^{n-1}\right) \cdot \nabla\right) \boldsymbol{u}^{n}+\left(\left(R_{h} \boldsymbol{u}^{n-1}-\boldsymbol{U}_{h}^{n-1}\right) \cdot \nabla\right) \boldsymbol{u}^{n} \\
& +\left(\left(\boldsymbol{U}_{h}^{n-1}-R_{h} \boldsymbol{u}^{n-1}\right) \cdot \nabla\right)\left(\boldsymbol{u}^{n}-R_{h} \boldsymbol{u}^{n}\right) \\
& +\left(\left(R_{h} \boldsymbol{u}^{n-1}-\boldsymbol{u}^{n-1}\right) \cdot \nabla\right)\left(\boldsymbol{u}^{n}-R_{h} \boldsymbol{u}^{n}\right) \\
& +\left(\boldsymbol{u}^{n-1} \cdot \nabla\right)\left(\boldsymbol{u}^{n}-R_{h} \boldsymbol{u}^{n}\right)+\left(\boldsymbol{U}_{h}^{n-1} \cdot \nabla\right)\left(R_{h} \boldsymbol{u}^{n}-\boldsymbol{U}_{h}^{n}\right) \\
:= & \sum_{k=1}^{6} \boldsymbol{B}_{k} .
\end{aligned}
$$

With the similar arguments as $A_{i}(i=1 \sim 4)$, we have

$$
\begin{aligned}
\left(\boldsymbol{B}_{1}, \boldsymbol{\theta}_{h}^{n}\right) & \leq C\left\|\boldsymbol{u}^{n-1}-R_{h} \boldsymbol{u}^{n-1}\right\|_{0}\left\|\nabla \boldsymbol{u}^{n}\right\|_{0, \infty}\left\|\boldsymbol{\theta}_{h}^{n}\right\|_{0} \\
& \leq C h^{2}\left(\left\|\boldsymbol{u}^{n-1}\right\|_{2}+\left\|p^{n-1}\right\|_{1}\right)\left\|\boldsymbol{\theta}_{h}^{n}\right\|_{0} \leq C h^{4}+C\left\|\boldsymbol{\theta}_{h}^{n}\right\|_{0}^{2}, \\
\left(\boldsymbol{B}_{2}, \boldsymbol{\theta}_{h}^{n}\right) & \leq C\left\|R_{h} \boldsymbol{u}^{n-1}-\boldsymbol{U}_{h}^{n-1}\right\|_{0}\left\|\nabla \boldsymbol{u}^{n}\right\|_{0, \infty}\left\|\boldsymbol{\theta}_{h}^{n}\right\|_{0} \\
& \leq C\left\|\boldsymbol{\theta}_{h}^{n-1}\right\|_{0}\left\|\boldsymbol{\theta}_{h}^{n}\right\|_{0} \leq C\left\|\boldsymbol{\theta}_{h}^{n-1}\right\|_{0}^{2}+C\left\|\boldsymbol{\theta}_{h}^{n}\right\|_{0}^{2}, \\
\left(\boldsymbol{B}_{3}, \boldsymbol{\theta}_{h}^{n}\right) & \leq\left\|\boldsymbol{U}_{h}^{n-1}-R_{h} \boldsymbol{u}^{n-1}\right\|_{0}\left\|\nabla\left(\boldsymbol{u}^{n}-R_{h} \boldsymbol{u}^{n}\right)\right\|_{0,2}\left\|\boldsymbol{\theta}_{h}^{n}\right\|_{0, \infty} \\
& \leq C\left\|\boldsymbol{\theta}_{h}^{n-1}\right\|_{0}\left(h\left(\left\|\boldsymbol{u}^{n}\right\|_{2}+\left\|p^{n}\right\|_{1}\right)\right)\left(h^{-1}\left\|\boldsymbol{\theta}_{h}^{n}\right\|_{0}\right) \\
& \leq C\left\|\boldsymbol{\theta}_{h}^{n-1}\right\|_{0}^{2}+C\left\|\boldsymbol{\theta}_{h}^{n}\right\|_{0}, \\
\left(\boldsymbol{B}_{4}, \boldsymbol{\theta}_{h}^{n}\right) & \leq C\left\|\boldsymbol{u}^{n-1}-R_{h} \boldsymbol{u}^{n-1}\right\|_{0}\left\|\nabla\left(\boldsymbol{u}^{n}-R_{h} \boldsymbol{u}^{n}\right)\right\|_{0}\left\|\boldsymbol{\theta}_{h}^{n}\right\|_{0, \infty} \\
& \leq C h^{2}\left(\left\|\boldsymbol{u}^{n-1}\right\|_{2}+\left\|p^{n-1}\right\|_{1}\right)\left(h\left(\left\|\boldsymbol{u}^{n}\right\|_{2}+\left\|p^{n}\right\|_{1}\right)\right)\left(h^{-1}\left\|\boldsymbol{\theta}_{h}^{n}\right\|_{0}\right) \\
& \leq C h^{4}+C\left\|\boldsymbol{\theta}_{h}^{n}\right\|_{0}^{2}, \\
\left(\boldsymbol{B}_{6}, \boldsymbol{\theta}_{h}^{n}\right) & \leq\left\|\boldsymbol{U}_{h}^{n-1}\right\|_{0, \infty}\left\|\nabla\left(R_{h} \boldsymbol{u}^{n}-\boldsymbol{U}_{h}^{n}\right)\right\|_{0}\left\|\boldsymbol{\theta}_{h}^{n}\right\|_{0} \\
& \leq C\left\|\nabla \boldsymbol{\theta}_{h}^{n}\right\|_{0}\left\|\boldsymbol{\theta}_{h}^{n}\right\|_{0} \leq C\left\|\boldsymbol{\theta}_{h}^{n}\right\|_{0}^{2}+\epsilon \nu\left\|\nabla \boldsymbol{\theta}_{h}^{n}\right\|_{0}^{2},
\end{aligned}
$$

and by applying Green's formula, we have

$$
\begin{aligned}
\left(\boldsymbol{B}_{5}, \boldsymbol{\theta}_{h}^{n}\right) & \left.=\left(\left(\boldsymbol{u}^{n-1} \cdot \nabla\right)\left(\boldsymbol{u}^{n}-R_{h} \boldsymbol{u}^{n}\right), \boldsymbol{\theta}_{h}^{n}\right)\right)=-\left(\left(\boldsymbol{u}^{n-1} \nabla\right) \boldsymbol{\theta}_{h}^{n}, \boldsymbol{u}^{n}-R_{h} \boldsymbol{u}^{n}\right) \\
& \leq\left\|\boldsymbol{u}^{n-1}\right\|_{0, \infty}\left\|\nabla \boldsymbol{\theta}_{h}^{n}\right\|_{0}\left\|\boldsymbol{u}^{n}-\boldsymbol{R}_{h} \boldsymbol{u}^{n}\right\|_{0} \\
& \leq C h^{2}\left(\left\|\boldsymbol{u}^{n}\right\|_{2}+\left\|p^{n}\right\|_{2}\right)\left\|\nabla \boldsymbol{\theta}_{h}^{n}\right\|_{0} \leq C h^{4}+\epsilon \nu\left\|\nabla \boldsymbol{\theta}_{h}^{n}\right\|_{0}^{2} .
\end{aligned}
$$


Based on the above estimates, we have

$$
\begin{aligned}
\left\|\boldsymbol{\theta}_{h}^{n}\right\|_{0}^{2}-\left\|\boldsymbol{\theta}_{h}^{n-1}\right\|_{0}^{2}+\nu \tau\left\|\nabla \boldsymbol{\theta}_{h}^{n}\right\|_{0}^{2} \leq & C \tau h^{4}+C \tau h^{4}\left(\left\|D_{\tau} \boldsymbol{u}^{n}\right\|_{2}^{2}+\left\|D_{\tau} p^{n}\right\|_{1}^{2}\right) \\
& +C \tau\left(\left\|\boldsymbol{\theta}_{h}^{n}\right\|_{0}^{2}+\left\|\boldsymbol{\theta}_{h}^{n-1}\right\|_{0}^{2}\right)+C \tau\left(\left\|\boldsymbol{R}_{1}^{n}\right\|_{0}^{2}+\left\|\boldsymbol{R}_{2}^{n}\right\|_{0}^{2}\right) .
\end{aligned}
$$

Summing up the above inequality leads to

$$
\begin{aligned}
& \left\|\boldsymbol{\theta}_{h}^{n}\right\|_{0}^{2}+\nu \tau \sum_{\ell=0}^{n}\left\|\nabla \boldsymbol{\theta}_{h}^{\ell}\right\|_{0}^{2} \\
\leq & C h^{4}+C h^{4} \tau \sum_{\ell=1}^{n}\left(\left\|D_{\tau} \boldsymbol{u}^{n}\right\|_{2}^{2}+\left\|D_{\tau} p^{n}\right\|_{1}^{2}\right)+C \tau \sum_{\ell=0}^{n}\left\|\boldsymbol{\theta}_{h}^{\ell}\right\|_{0}^{2}+C \tau \sum_{\ell=1}^{n}\left(\left\|\boldsymbol{R}_{1}^{n}\right\|_{0}^{2}+\left\|\boldsymbol{R}_{2}^{n}\right\|_{0}^{2}\right) \\
\leq & C \tau \sum_{\ell=0}^{n}\left\|\boldsymbol{\theta}_{h}^{\ell}\right\|_{0}^{2}+C\left(h^{4}+\tau^{2}\right),
\end{aligned}
$$

where we have used $\left\|\boldsymbol{\theta}_{h}^{0}\right\|_{0}=\left\|R_{h} \boldsymbol{u}_{0}-\Pi_{h} \boldsymbol{u}_{0}\right\|_{0} \leq C h^{2}\left\|\boldsymbol{u}_{0}\right\|_{2}$.

Thus, thanks to Lemma 2.2, there exist a small $\tau_{4}$, when $\tau \leq \tau_{4}$, such that

$$
\left\|\boldsymbol{\theta}_{h}^{n}\right\|_{0}^{2}+\nu \tau \sum_{\ell=0}^{n}\left\|\nabla \boldsymbol{\theta}_{h}^{\ell}\right\|_{0}^{2} \leq C\left(h^{4}+\tau^{2}\right)
$$

Secondly, we focus on the error estimate of $\left\|\nabla \boldsymbol{\theta}_{h}^{n}\right\|_{0}$. To do this, by taking $\boldsymbol{v}_{h}=2 \tau D_{\tau} \boldsymbol{\theta}_{h}^{n}$ in (5.3) and $q_{h}=\eta_{h}^{n}$ in (5.4), we arrive at

$$
\begin{aligned}
& 2 \tau\left\|D_{\tau} \boldsymbol{\theta}_{h}^{n}\right\|_{0}^{2}+\nu\left(\left\|\nabla \boldsymbol{\theta}_{h}^{n}\right\|_{0}^{2}-\left\|\nabla \boldsymbol{\theta}_{h}^{n-1}\right\|_{0}^{2}\right) \\
\leq & -\left(D_{\tau}\left(\boldsymbol{u}^{n}-R_{h} \boldsymbol{u}^{n}\right), 2 \tau D_{\tau} \boldsymbol{\theta}_{h}^{n}\right)-\left(\left(\boldsymbol{u}^{n-1} \cdot \nabla\right) \boldsymbol{u}^{n}-\left(\boldsymbol{U}_{h}^{n-1} \cdot \nabla\right) \boldsymbol{U}_{h}^{n-1}, 2 \tau D_{\tau} \boldsymbol{\theta}_{h}^{n}\right) \\
& +\left(\boldsymbol{R}_{1}^{n}, 2 \tau D_{\tau} \boldsymbol{\theta}_{h}^{n}\right)+\left(\boldsymbol{R}_{2}^{n}, 2 \tau D_{\tau} \boldsymbol{\theta}_{h}^{n}\right) .
\end{aligned}
$$

It is easy to see that

$$
\begin{aligned}
\left(D_{\tau}\left(\boldsymbol{u}^{n}-R_{h} \boldsymbol{u}^{n}\right), 2 \tau D_{\tau} \boldsymbol{\theta}_{h}^{n}\right) & \leq C \tau\left\|D_{\tau}\left(\boldsymbol{u}^{n}-R_{h} \boldsymbol{u}^{n}\right)\right\|_{0}\left\|D_{\tau} \boldsymbol{\theta}_{h}^{n}\right\|_{0} \\
& \leq C \tau h\left(\left\|D_{\tau} \boldsymbol{u}^{n}\right\|_{2}+\left\|D_{\tau} p^{n}\right\|_{1}\right)\left\|D_{\tau} \boldsymbol{\theta}_{h}^{n}\right\|_{0} \\
& \leq C \tau h^{2}\left(\left\|D_{\tau} \boldsymbol{u}^{n}\right\|_{2}^{2}+\left\|D_{\tau} p^{n}\right\|_{1}^{2}\right)+\epsilon \tau\left\|D_{\tau} \boldsymbol{\theta}_{h}^{n}\right\|_{0}^{2}
\end{aligned}
$$

and

$$
\begin{aligned}
\left(\boldsymbol{R}_{1}^{n}, 2 \tau D_{\tau} \boldsymbol{\theta}_{h}^{n}\right)+\left(\boldsymbol{R}_{2}^{n}, 2 \tau D_{\tau} \boldsymbol{\theta}_{h}^{n}\right) & \leq C \tau\left(\left\|\boldsymbol{R}_{1}^{n}\right\|_{0}+\left\|\boldsymbol{R}_{2}^{n}\right\|_{0}\right)\left\|D_{\tau} \boldsymbol{\theta}_{h}^{n}\right\|_{0} \\
& \leq C \tau\left(\left\|\boldsymbol{R}_{1}^{n}\right\|_{0}^{2}+\left\|\boldsymbol{R}_{2}^{n}\right\|_{0}^{2}\right)+\epsilon \tau\left\|D_{\tau} \boldsymbol{\theta}_{h}^{n}\right\|_{0}^{2} .
\end{aligned}
$$

Moreover, we have

$$
\begin{aligned}
\left(\boldsymbol{B}_{1}, 2 \tau D_{\tau} \boldsymbol{\theta}_{h}^{n}\right) & \leq C \tau\left\|\boldsymbol{u}^{n-1}-R_{h} \boldsymbol{u}^{n-1}\right\|_{0}\left\|\nabla \boldsymbol{u}^{n}\right\|_{0, \infty}\left\|D_{\tau} \boldsymbol{\theta}_{h}^{n}\right\|_{0} \\
& \leq C \tau h\left(\left\|\boldsymbol{u}^{n-1}\right\|_{2}+\left\|p^{n-1}\right\|_{1}\right)\left\|D_{\tau} \boldsymbol{\theta}_{h}^{n}\right\|_{0} \leq C \tau h^{2}+\epsilon \tau\left\|D_{\tau} \boldsymbol{\theta}_{h}^{n}\right\|_{0}^{2}, \\
\left(\boldsymbol{B}_{2}, 2 \tau D_{\tau} \boldsymbol{\theta}_{h}^{n}\right) & \leq C \tau\left\|R_{h} \boldsymbol{u}^{n-1}-\boldsymbol{U}_{h}^{n-1}\right\|_{0}\left\|\nabla \boldsymbol{u}^{n}\right\|_{0, \infty}\left\|D_{\tau} \boldsymbol{\theta}_{h}^{n}\right\|_{0} \\
& \leq C \tau\left\|\nabla \boldsymbol{\theta}_{h}^{n}\right\|_{0}\left\|D_{\tau} \boldsymbol{\theta}_{h}^{n}\right\|_{0} \leq C \tau\left\|\nabla \boldsymbol{\theta}_{h}^{n}\right\|_{0}^{2}+\epsilon \tau\left\|D_{\tau} \boldsymbol{\theta}_{h}^{n}\right\|_{0}^{2}, \\
\left(\boldsymbol{B}_{3}, 2 \tau D_{\tau} \boldsymbol{\theta}_{h}^{n}\right) & \leq C \tau\left\|\boldsymbol{U}_{h}^{n-1}-R_{h} \boldsymbol{u}^{n-1}\right\|_{0}\left\|\nabla\left(\boldsymbol{u}^{n}-R_{h} \boldsymbol{u}^{n}\right)\right\|_{0}\left\|D_{\tau} \boldsymbol{\theta}_{h}^{n}\right\|_{0, \infty} \\
& \leq C \tau\left\|\boldsymbol{\theta}_{h}^{n-1}\right\|_{0}\left(h\left(\left\|\boldsymbol{u}^{n}\right\|_{2}+\left\|p^{n}\right\|_{1}\right)\right)\left(h^{-1}\left\|D_{\tau} \boldsymbol{\theta}_{h}^{n}\right\|_{0}\right) \\
& \leq C \tau\left\|\nabla \boldsymbol{\theta}_{h}^{n-1}\right\|_{0}\left\|D_{\tau} \boldsymbol{\theta}_{h}^{n}\right\|_{0} \leq C \tau\left\|\nabla \boldsymbol{\theta}_{h}^{n-1}\right\|_{0}^{2}+\epsilon \tau\left\|D_{\tau} \boldsymbol{\theta}_{h}^{n}\right\|_{0}^{2},
\end{aligned}
$$




$$
\begin{aligned}
\left(\boldsymbol{B}_{4}, 2 \tau D_{\tau} \boldsymbol{\theta}_{h}^{n}\right) & \leq C \tau\left\|R_{h} \boldsymbol{u}^{n-1}-\boldsymbol{u}^{n-1}\right\|_{0}\left\|\nabla\left(\boldsymbol{u}^{n}-R_{h} \boldsymbol{u}^{n}\right)\right\|_{0}\left\|D_{\tau} \boldsymbol{\theta}_{h}^{n}\right\|_{0, \infty} \\
& \leq C \tau\left(h^{2}\left(\left\|\boldsymbol{u}^{n-1}\right\|_{2}+\left\|p^{n-1}\right\|_{1}\right)\right)\left(h\left(\left\|\boldsymbol{u}^{n}\right\|_{2}+\left\|p^{n}\right\|_{1}\right)\right)\left(h^{-1}\left\|D_{\tau} \boldsymbol{\theta}_{h}^{n}\right\|_{0}\right) \\
& \leq C \tau h\left\|D_{\tau} \boldsymbol{\theta}_{h}^{n}\right\|_{0} \leq C \tau h^{2}+\epsilon \tau\left\|D_{\tau} \boldsymbol{\theta}_{h}^{n}\right\|_{0}^{2}, \\
\left(\boldsymbol{B}_{5}, 2 \tau D_{\tau} \boldsymbol{\theta}_{h}^{n}\right) & \leq C \tau\left\|\boldsymbol{u}^{n-1}\right\|_{0, \infty}\left\|\nabla\left(\boldsymbol{u}^{n}-\boldsymbol{R}_{h} \boldsymbol{u}^{n}\right)\right\|_{0}\left\|D_{\tau} \boldsymbol{\theta}_{h}^{n}\right\|_{0} \\
& \leq C \tau\left(h\left(\left\|\boldsymbol{u}^{n}\right\|_{2}+\left\|p^{n}\right\|_{1}\right)\right)\left\|D_{\tau} \boldsymbol{\theta}_{h}^{n}\right\|_{0} \\
& \leq C \tau h\left\|D_{\tau} \boldsymbol{\theta}_{h}^{n}\right\|_{0} \leq C \tau h^{2}+\epsilon \tau\left\|D_{\tau} \boldsymbol{\theta}_{h}^{n}\right\|_{0}^{2}, \\
\left(\boldsymbol{B}_{6}, 2 \tau D_{\tau} \boldsymbol{\theta}_{h}^{n}\right) & \leq C \tau\left\|\boldsymbol{U}_{h}^{n-1}\right\|_{0, \infty}\left\|\nabla\left(\boldsymbol{R}_{h} \boldsymbol{u}^{n}-\boldsymbol{U}_{h}^{n}\right)\right\|_{0}\left\|D_{\tau} \boldsymbol{\theta}_{h}^{n}\right\|_{0} \\
& \leq C \tau\left\|\nabla \boldsymbol{\theta}_{h}^{n}\right\|_{0}\left\|D_{\tau} \boldsymbol{\theta}_{h}^{n}\right\|_{0} \leq C \tau\left\|\nabla \boldsymbol{\theta}_{h}^{n}\right\|_{0}^{2}+\epsilon \tau\left\|D_{\tau} \boldsymbol{\theta}_{h}^{n}\right\|_{0}^{2} .
\end{aligned}
$$

Based on the above estimates, we deduce

$$
\begin{aligned}
\left\|\nabla \boldsymbol{\theta}_{h}^{n}\right\|_{0}^{2}-\left\|\nabla \boldsymbol{\theta}_{h}^{n-1}\right\|_{0}^{2}+\tau\left\|D_{\tau} \boldsymbol{\theta}_{h}^{n}\right\|_{0}^{2} \leq & C \tau h^{2}+C \tau h^{2}\left(\left\|D_{\tau} \boldsymbol{u}^{n}\right\|_{2}^{2}+\left\|D_{\tau} p^{n}\right\|_{1}^{2}\right) \\
& +C \tau\left(\left\|\nabla \boldsymbol{\theta}_{h}^{n}\right\|_{0}^{2}+\left\|\nabla \boldsymbol{\theta}_{h}^{n-1}\right\|_{0}^{2}\right)+C \tau\left(\left\|\boldsymbol{R}_{1}^{n}\right\|_{0}^{2}+\left\|\boldsymbol{R}_{2}^{n}\right\|_{0}^{2}\right) .
\end{aligned}
$$

Summing up the above inequality leads to

$$
\begin{aligned}
\left\|\nabla \boldsymbol{\theta}_{h}^{n}\right\|_{0}^{2}+\tau \sum_{\ell=1}^{n}\left\|D_{\tau} \boldsymbol{\theta}_{h}^{\ell}\right\|_{0}^{2} \leq & C h^{2}+C h^{2} \tau \sum_{\ell=1}^{n}\left(\left\|D_{\tau} \boldsymbol{u}^{n}\right\|_{2}^{2}+\left\|D_{\tau} p^{n}\right\|_{1}^{2}\right) \\
& +C \tau \sum_{\ell=0}^{n}\left\|\nabla \boldsymbol{\theta}_{h}^{\ell}\right\|_{0}^{2}+C \tau \sum_{\ell=1}^{n}\left(\left\|\boldsymbol{R}_{1}^{n}\right\|_{0}^{2}+\left\|\boldsymbol{R}_{2}^{n}\right\|_{0}^{2}\right) \\
\leq & C \tau \sum_{\ell=0}^{n}\left\|\nabla \boldsymbol{\theta}_{h}^{\ell}\right\|_{0}^{2}+C\left(h^{2}+\tau^{2}\right) .
\end{aligned}
$$

According to Lemma 2.2, there exists a small $\tau_{5}$, when $\tau \leq \tau_{5}$, such that

$$
\left\|\nabla \boldsymbol{\theta}_{h}^{n}\right\|_{0}^{2}+\tau \sum_{\ell=1}^{n}\left\|D_{\tau} \boldsymbol{\theta}_{h}^{\ell}\right\|_{0}^{2} \leq C\left(h^{2}+\tau^{2}\right) .
$$

Finally, we present the error estimate of $\left\|\eta_{h}^{n}\right\|_{0}$. To do this, rewrite (5.3) by

$$
\begin{aligned}
\left(\eta_{h}^{n}, \nabla \cdot \boldsymbol{v}_{h}\right)= & -\left(D_{\tau} \boldsymbol{\theta}_{h}^{n}, \boldsymbol{v}_{h}\right)-\nu\left(\nabla \boldsymbol{\theta}_{h}^{n}, \boldsymbol{v}_{h}\right)+\left(D_{\tau}\left(\boldsymbol{u}^{n}-R_{h} \boldsymbol{u}^{n}\right), \boldsymbol{v}_{h}\right) \\
& +\left(\left(\boldsymbol{u}^{n-1} \cdot \nabla\right) \boldsymbol{u}^{n}-\left(\boldsymbol{U}_{h}^{n-1} \cdot \nabla\right) \boldsymbol{U}_{h}^{n}, \boldsymbol{v}_{h}\right)-\left(\boldsymbol{R}_{1}^{n}, \boldsymbol{v}_{h}\right)-\left(\boldsymbol{R}_{2}^{n}, \boldsymbol{v}_{h}\right) .
\end{aligned}
$$

Then, we can see that

$$
\begin{aligned}
\left(\eta_{h}^{n}, \nabla \cdot \boldsymbol{v}_{h}\right) \leq & C\left(\left\|D_{\tau} \boldsymbol{\theta}_{h}^{n}\right\|_{0}+\left\|\nabla \boldsymbol{\theta}_{h}^{n}\right\|_{0}+\left\|D_{\tau}\left(\boldsymbol{u}^{n}-R_{h} \boldsymbol{u}^{n}\right)\right\|_{0}\right. \\
& \left.+\left\|\left(\boldsymbol{u}^{n-1} \cdot \nabla\right) \boldsymbol{u}^{n}-\left(\boldsymbol{U}_{h}^{n-1} \cdot \nabla\right) \boldsymbol{U}_{h}^{n}\right\|_{0}+\left\|\boldsymbol{R}_{1}^{n}\right\|_{0}+\left\|\boldsymbol{R}_{2}^{n}\right\|_{0}\right)\left\|\nabla \boldsymbol{v}_{h}\right\|_{0} .
\end{aligned}
$$

By using discrete LBB condition (2.4) and the estimates obtained above, we get

$$
\begin{aligned}
\left\|\eta_{h}^{n}\right\|_{0} \leq & C \sup _{0 \neq \boldsymbol{v}_{h} \in \boldsymbol{V}_{h}} \frac{\left(\eta_{h}^{n}, \nabla \cdot \boldsymbol{v}_{h}\right)}{\left\|\nabla \boldsymbol{v}_{h}\right\|_{0}} \\
\leq & C\left(\left\|D_{\tau} \boldsymbol{\theta}_{h}^{n}\right\|_{0}+\left\|\nabla \boldsymbol{\theta}_{h}^{n}\right\|_{0}+\left\|D_{\tau}\left(\boldsymbol{u}^{n}-R_{h} \boldsymbol{u}^{n}\right)\right\|_{0}\right. \\
& \left.+\left\|\left(\boldsymbol{u}^{n-1} \cdot \nabla\right) \boldsymbol{u}^{n}-\left(\boldsymbol{U}_{h}^{n-1} \cdot \nabla\right) \boldsymbol{U}_{h}^{n}\right\|_{0}+\left\|\boldsymbol{R}_{1}^{n}\right\|_{0}+\left\|\boldsymbol{R}_{2}^{n}\right\|_{0}\right) \\
\leq & C\left(\left\|D_{\tau} \boldsymbol{\theta}_{h}^{n}\right\|_{0}+\left\|\boldsymbol{R}_{1}^{n}\right\|_{0}+\left\|\boldsymbol{R}_{2}^{n}\right\|_{0}+h+\tau\right)
\end{aligned}
$$


by $(5.27)$ and $(3.10)$, it follows that

$$
\begin{aligned}
\tau \sum_{\ell=1}^{N}\left\|\eta_{h}^{n}\right\|_{0}^{2} & \leq C\left(\tau \sum_{\ell=1}^{N}\left\|D_{\tau} \boldsymbol{\theta}_{h}^{\ell}\right\|_{0}^{2}+\tau \sum_{\ell=0}^{N}\left(\left\|\boldsymbol{R}_{1}^{n}\right\|_{0}^{2}+\left\|\boldsymbol{R}_{2}^{n}\right\|_{0}^{2}\right)+h^{2}+\tau^{2}\right) \\
& \leq C\left(h^{2}+\tau^{2}\right) .
\end{aligned}
$$

Taking $\tau_{0}=\min \left\{\tau^{* *}, \tau_{4}, \tau_{5}\right\}$ and $h_{0} \leq h_{1}$, the proof of Theorem 2.1 is complete by the $(2.7)$ and triangle inequality.

\section{Numerical Results}

In this section, some numerical results are provided to confirm the theoretical analysis. The final time is set $T=1.0$ in all computations and the domain $\Omega=(0,1)^{2}$. A regular triangulation with $M+1$ nodes in both horizontal and vertical directions is made for the domain $\Omega$.

Example 6.1. The viscosity coefficient $\nu=1$, the boundary/initial conditions and the source term $\boldsymbol{f}$ are chosen according to the exact solutions

$$
\begin{aligned}
& u_{1}=-e^{-2 t} \sin ^{2}\left(\pi x_{1}\right) \sin \left(\pi x_{2}\right) \cos \left(\pi x_{2}\right), \quad u_{2}=e^{-2 t} \sin \left(\pi x_{1}\right) \cos \left(\pi x_{1}\right) \sin ^{2}\left(\pi x_{2}\right), \\
& p=e^{-4 t} \sin \left(\pi x_{1}\right) \cos \left(\pi x_{2}\right) .
\end{aligned}
$$

In order to demonstrate the error estimates in Theorems 2.1, we choose $\tau=\mathcal{O}\left(h^{2}\right)$ and present the numerical results with respect to $t=0.2,0.6,1.0$ in Tables 6.1-6.3, respectively. It can been see that the convergence rates for the velocity $\boldsymbol{u}$ are in good agreement with the theoretical analysis. The convergence rates for the pressure $p$ in $L^{\infty} \times L^{2}$-norm are also included in Tables 6.1-6.3, although the theoretical analysis was given only in the $L^{2} \times L^{2}$-norm. For clarity, we also present the graphics of exact solutions and numerical solutions Figs. 6.1-6.3 at

Table 6.1: The numerical errors at $t=0.2$ of Example 6.1.

\begin{tabular}{|lllll|}
\hline$M \times M$ & $8 \times 8$ & $16 \times 16$ & $32 \times 32$ & $64 \times 64$ \\
\hline$\left\|\boldsymbol{u}^{n}-\boldsymbol{U}_{h}^{n}\right\|_{0}$ & $8.4849 \mathrm{e}-03$ & $2.6682 \mathrm{e}-03$ & $5.1766 \mathrm{e}-04$ & $1.6655 \mathrm{e}-04$ \\
Rate & $/$ & 1.6690 & 2.3658 & 1.6360 \\
$\left\|\boldsymbol{u}^{n}-\boldsymbol{U}_{h}^{n}\right\|_{1}$ & $3.3903 \mathrm{e}-01$ & $1.6877 \mathrm{e}-01$ & $8.4426 \mathrm{e}-02$ & $4.2201 \mathrm{e}-02$ \\
Rate & $/$ & 1.0063 & 0.99933 & 1.0004 \\
$\left\|p^{n}-P_{h}^{n}\right\|_{0}$ & $4.6940 \mathrm{e}-02$ & $1.9257 \mathrm{e}-02$ & $9.2024 \mathrm{e}-03$ & $4.5227 \mathrm{e}-03$ \\
Rate & $/$ & 1.2854 & 1.0653 & 1.0248 \\
\hline
\end{tabular}

Table 6.2: The numerical errors at $t=0.6$ of Example 6.1.

\begin{tabular}{|lllll|}
\hline$M \times M$ & $8 \times 8$ & $16 \times 16$ & $32 \times 32$ & $64 \times 64$ \\
\hline$\left\|\boldsymbol{u}^{n}-\boldsymbol{U}_{h}^{n}\right\|_{0}$ & $4.4365 \mathrm{e}-03$ & $1.0105 \mathrm{e}-03$ & $2.7426 \mathrm{e}-04$ & $6.2876 \mathrm{e}-05$ \\
Rate & $/$ & 2.1343 & 1.8815 & 2.1250 \\
$\left\|\boldsymbol{u}^{n}-\boldsymbol{U}_{h}^{n}\right\|_{1}$ & $1.5172 \mathrm{e}-01$ & $7.5884 \mathrm{e}-02$ & $3.7925 \mathrm{e}-02$ & $1.8963 \mathrm{e}-02$ \\
Rate & $/$ & 0.99955 & 1.0007 & 0.99997 \\
$\left\|p^{n}-P_{h}^{n}\right\|_{0}$ & $1.3955 \mathrm{e}-02$ & $4.7630 \mathrm{e}-03$ & $1.9727 \mathrm{e}-03$ & $9.2927 \mathrm{e}-04$ \\
Rate & $/$ & 1.5509 & 1.2717 & 1.0860 \\
\hline
\end{tabular}


Table 6.3: The numerical errors at $t=1.0$ of Example 6.1.

\begin{tabular}{|lllll|}
\hline$M \times M$ & $8 \times 8$ & $16 \times 16$ & $32 \times 32$ & $64 \times 64$ \\
\hline$\left\|\boldsymbol{u}^{n}-\boldsymbol{U}_{h}^{n}\right\|_{0}$ & $2.3455 \mathrm{e}-03$ & $5.8688 \mathrm{e}-04$ & $1.4674 \mathrm{e}-04$ & $3.6687 \mathrm{e}-05$ \\
Rate & $/$ & 1.9988 & 1.9998 & 1.9999 \\
$\left\|\boldsymbol{u}^{n}-\boldsymbol{U}_{h}^{n}\right\|_{1}$ & $6.8101 \mathrm{e}-02$ & $3.4073 \mathrm{e}-02$ & $1.7040 \mathrm{e}-02$ & $8.5202 \mathrm{e}-03$ \\
Rate & $/$ & 0.99903 & 0.99975 & 0.99994 \\
$\left\|p^{n}-P_{h}^{n}\right\|_{0}$ & $5.4383 \mathrm{e}-03$ & $1.5399 \mathrm{e}-03$ & $5.0090 \mathrm{e}-04$ & $2.0240 \mathrm{e}-04$ \\
Rate & $/$ & 1.8203 & 1.6202 & 1.3073 \\
\hline
\end{tabular}

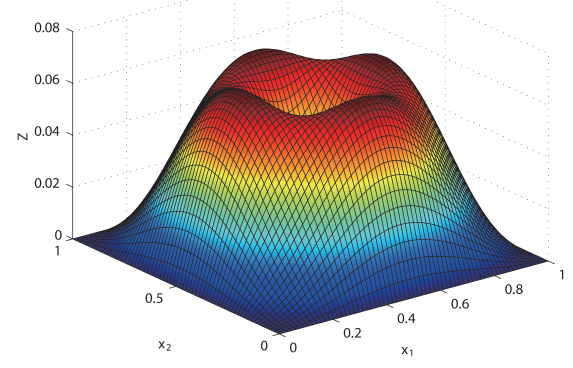

(a) $\left|\boldsymbol{u}^{n}\right|$.

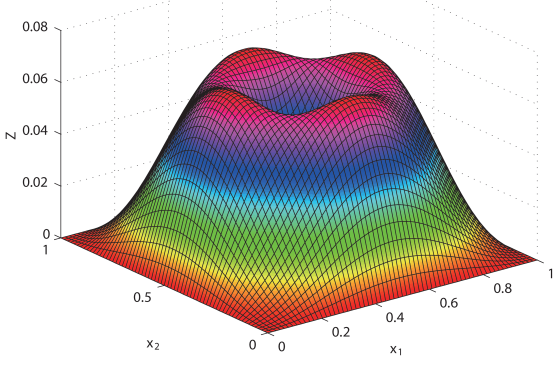

(b) $\left|\boldsymbol{U}_{h}^{n}\right|$.

Fig. 6.1. The graphics at $t=1.0$ on mesh $64 \times 64$ of Example 6.1.

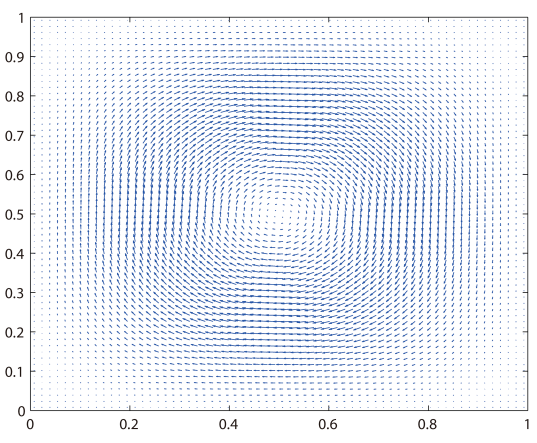

(a) The vector field of $\boldsymbol{u}^{n}$.

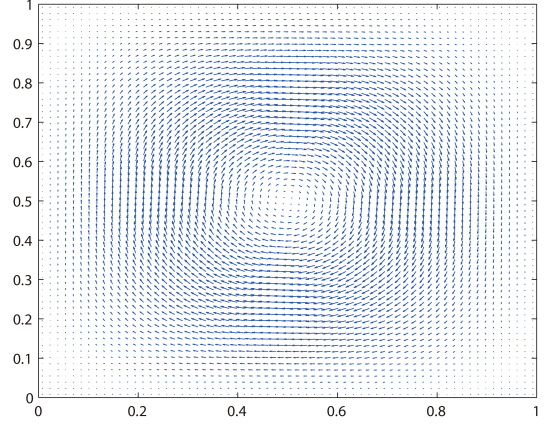

(b) The vector field of $\boldsymbol{U}_{h}^{n}$.

Fig. 6.2. The graphics at $t=1.0$ on mesh $64 \times 64$ of Example 6.1.

$t=1.0$. These also indicate that the numerical results are in accordance with the theoretical analysis.

On the other hand, to show the unconditional convergence of the linearized Euler scheme (2.2)-(2.3), we use different time steps $\tau=0.1,0.05,0.01$ on gradually refined meshes $M=$ $10 i, i=1, \ldots, 8$ at $t=1.0$. The $L^{2}$-norm, $H^{1}$-norm for the velocity and $L^{2}$-norm for the pressure are given in Fig. 6.4. It is easy to see that the scheme is stable for large time steps, which indicate that the time step restriction is not necessary. 


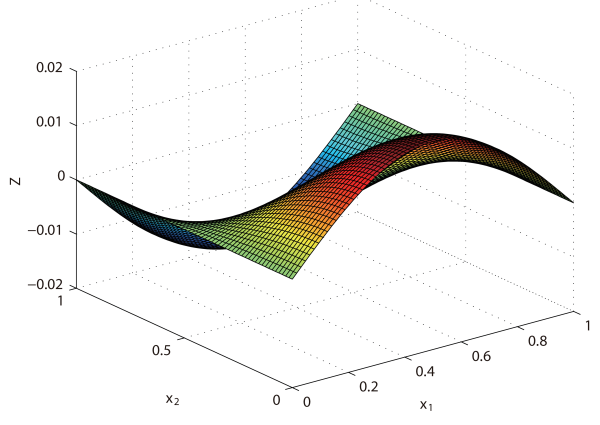

(a) Exact solution $p^{n}$.

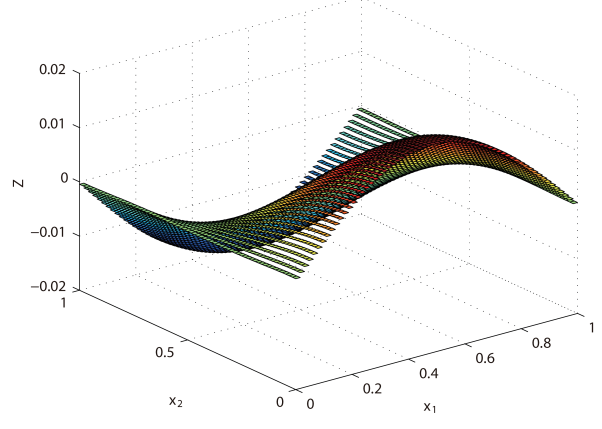

(b) Numerical solution $P_{h}^{n}$.

Fig. 6.3. The graphics at $t=1.0$ on mesh $64 \times 64$ of Example 6.1.

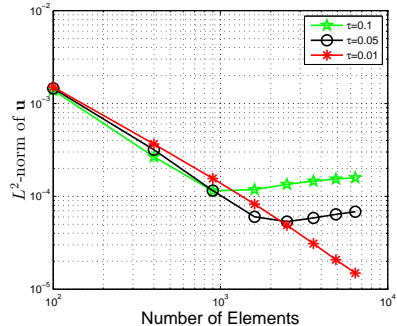

(a) $L^{2}$-norm of $\boldsymbol{u}$.

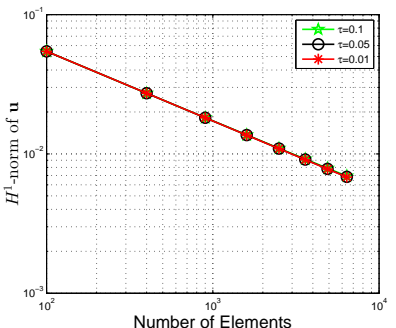

(b) $H^{1}$-norm of $\boldsymbol{u}$.

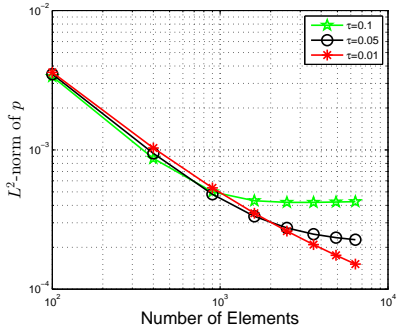

(c) $L^{2}$-norm of $p$.

Fig. 6.4. The error of different time-steps.

Example 6.2. The viscosity coefficient $\nu=0.01$, the boundary/initial conditions and the source term $\boldsymbol{f}$ are chosen according to the exact solutions

$$
\begin{aligned}
& u_{1}=e^{-t}\left(x_{1}^{4}-2 x_{1}^{3}+x_{1}^{2}\right)\left(4 x_{2}^{3}-6 x_{2}^{2}+2 x_{2}\right), \\
& u_{2}=-e^{-t}\left(x_{2}^{4}-2 x_{2}^{3}+x_{2}^{2}\right)\left(4 x_{1}^{3}-6 x_{1}^{2}+2 x_{1}\right), \\
& p=10 e^{-t}\left(2 x_{1}-1\right)\left(2 x_{2}-1\right) .
\end{aligned}
$$

We present the numerical results with respect to $t=0.2,0.6,1.0$ in Tables 6.4-6.6, respectively. It can been seen from Tables 6.4-6.6 that the numerical results are in accordance with the theoretical analysis. For clarity, we also present the graphics of exact solutions and numerical solutions at $t=1.0$ in Figs. 6.5-6.7, respectively. Moreover, we use different time steps $\tau=$

Table 6.4: The numerical errors at $t=0.2$ of Example 6.2.

\begin{tabular}{|lllll|}
\hline$M \times M$ & $8 \times 8$ & $16 \times 16$ & $32 \times 32$ & $64 \times 64$ \\
\hline$\left\|\boldsymbol{u}^{n}-\boldsymbol{U}_{h}^{n}\right\|_{0}$ & $5.3019 \mathrm{e}-04$ & $1.4750 \mathrm{e}-04$ & $3.4029 \mathrm{e}-05$ & $9.2819 \mathrm{e}-06$ \\
Rate & $/$ & 1.8458 & 2.1159 & 1.8743 \\
$\left\|\boldsymbol{u}^{n}-\boldsymbol{U}_{h}^{n}\right\|_{1}$ & $1.2641 \mathrm{e}-02$ & $6.3238 \mathrm{e}-03$ & $3.1586 \mathrm{e}-03$ & $1.5793 \mathrm{e}-03$ \\
Rate & $/$ & 0.99925 & 1.0015 & 1.0000 \\
$\left\|p^{n}-P_{h}^{n}\right\|_{0}$ & $4.9843 \mathrm{e}-01$ & $2.4311 \mathrm{e}-01$ & $1.2086 \mathrm{e}-01$ & $6.0335 \mathrm{e}-02$ \\
Rate & $/$ & 1.0358 & 1.0082 & 1.0023 \\
\hline
\end{tabular}


Table 6.5: The numerical errors at $t=0.6$ of Example 6.2.

\begin{tabular}{|lllll|}
\hline$M \times M$ & $8 \times 8$ & $16 \times 16$ & $32 \times 32$ & $64 \times 64$ \\
\hline$\left\|\boldsymbol{u}^{n}-\boldsymbol{U}_{h}^{n}\right\|_{0}$ & $4.6361 \mathrm{e}-04$ & $1.1624 \mathrm{e}-04$ & $2.9987 \mathrm{e}-05$ & $7.3266 \mathrm{e}-06$ \\
Rate & $/$ & 1.9958 & 1.9547 & 2.0331 \\
$\left\|\boldsymbol{u}^{n}-\boldsymbol{U}_{h}^{n}\right\|_{1}$ & $8.5993 \mathrm{e}-03$ & $4.2505 \mathrm{e}-03$ & $2.1195 \mathrm{e}-03$ & $1.0588 \mathrm{e}-03$ \\
Rate & $/$ & 1.0166 & 1.0039 & 1.0013 \\
$\left\|p^{n}-P_{h}^{n}\right\|_{0}$ & $3.3353 \mathrm{e}-01$ & $1.6301 \mathrm{e}-01$ & $8.1008 \mathrm{e}-02$ & $4.0444 \mathrm{e}-02$ \\
Rate & $/$ & 1.0329 & 1.0088 & 1.0021 \\
\hline
\end{tabular}

Table 6.6: The numerical errors at $t=1.0$ of Example 6.2.

\begin{tabular}{|lllll|}
\hline$M \times M$ & $8 \times 8$ & $16 \times 16$ & $32 \times 32$ & $64 \times 64$ \\
\hline$\left\|\boldsymbol{u}^{n}-\boldsymbol{U}_{h}^{n}\right\|_{0}$ & $3.9818 \mathrm{e}-04$ & $1.0275 \mathrm{e}-04$ & $2.5888 \mathrm{e}-05$ & $6.4845 \mathrm{e}-06$ \\
Rate & $/$ & 1.9543 & 1.9888 & 1.9972 \\
$\left\|\boldsymbol{u}^{n}-\boldsymbol{U}_{h}^{n}\right\|_{1}$ & $5.9408 \mathrm{e}-03$ & $2.8750 \mathrm{e}-03$ & $1.4238 \mathrm{e}-03$ & $7.1016 \mathrm{e}-04$ \\
Rate & $/$ & 1.0471 & 1.0138 & 1.0036 \\
$\left\|p^{n}-P_{h}^{n}\right\|_{0}$ & $2.2345 \mathrm{e}-01$ & $1.0923 \mathrm{e}-01$ & $5.4300 \mathrm{e}-02$ & $2.7110 \mathrm{e}-02$ \\
Rate & $/$ & 1.0325 & 1.0084 & 1.0021 \\
\hline
\end{tabular}

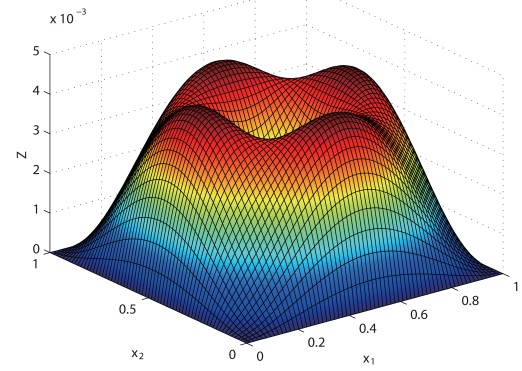

(a) $\left|\boldsymbol{u}^{n}\right|$

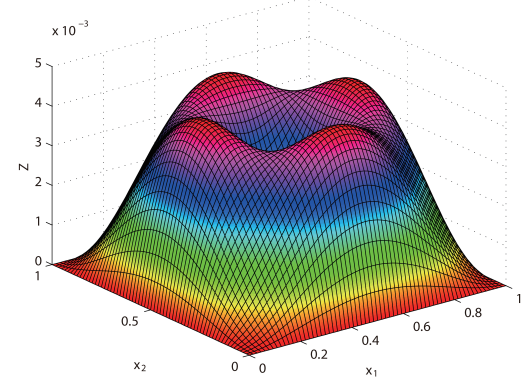

(b) $\left|\boldsymbol{U}_{h}^{n}\right|$

Fig. 6.5. The graphics at $t=1.0$ on mesh $64 \times 64$ of Example 6.2

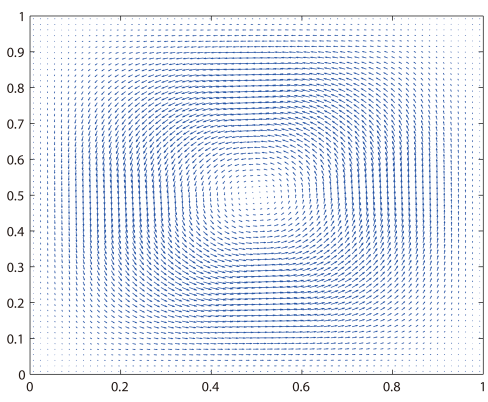

(a) The vector field of $\boldsymbol{u}^{n}$

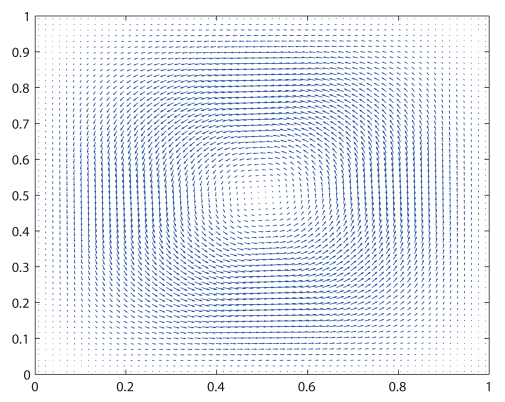

(b) The vector field of $\boldsymbol{U}_{h}^{n}$

Fig. 6.6. The graphics at $t=1.0$ on mesh $64 \times 64$ of Example 6.2. 


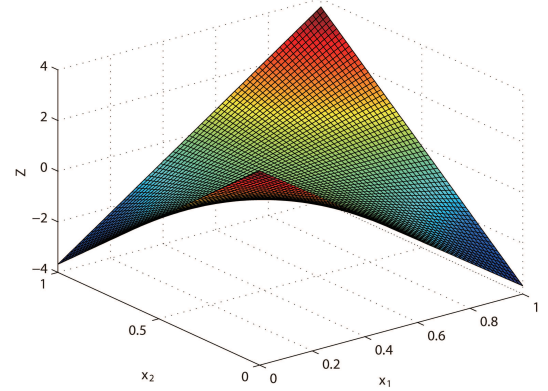

(a) Exact solution $p^{n}$

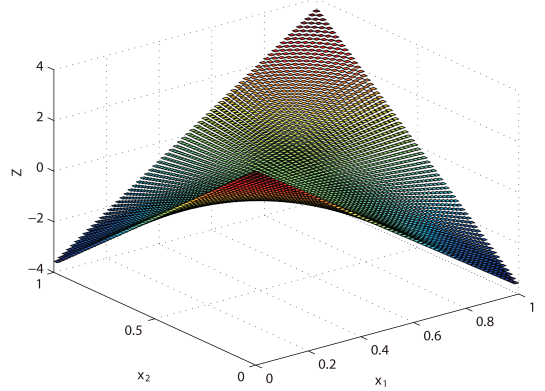

(b) Numerical solution $P_{h}^{n}$

Fig. 6.7. The graphics at $t=1.0$ on mesh $64 \times 64$ of Example 6.2 .

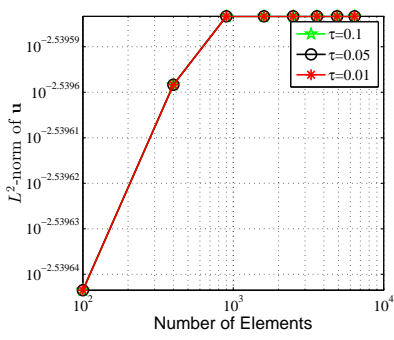

(a) $L^{2}$-norm of $\boldsymbol{u}$.

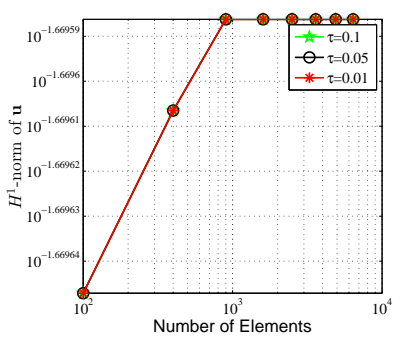

(b) $H^{1}$-norm of $\boldsymbol{u}$.

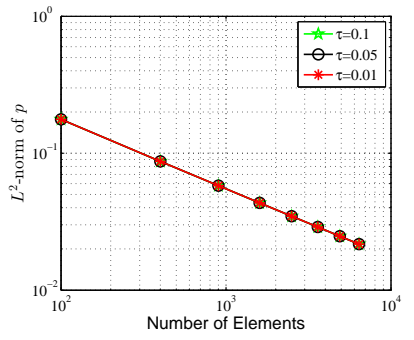

(c) $L^{2}$-norm of $p$.

Fig. 6.8. The error of different time-steps.

$0.1,0.05,0.01$ on gradually refined meshes $M=10 i, i=1,2, \ldots, 8$ at $t=1.0$ to demonstrate the unconditional stability of the linearized Euler scheme (2.2)-(2.3) and graph the results in Fig. 6.8.

Acknowledgements. This work is supported by National Natural Science Foundation of China (No. 11671369) and the Doctoral Starting Foundation of Zhengzhou University of Aeronautics (No. 63020390).

\section{References}

[1] V. Girault, P.A. Raviart, Finite element method for Navier-Stokes equations: Theory and Algorithms, Springer-Verlag, Berlin, 1987.

[2] R. Teman, Navier-Stokes equations: Theory and Numerical analysis, North Holland, AmsterdamNew York-Oxford, 1977.

[3] Y. He, W. Sun, Sability and convergence of the Crank-Nicolson/Adams-Bashforth scheme for the time-dependent Navier-Stokes equations, SIAM J. Numer. Anal., 45 (2007), 837-869.

[4] J. G. Heywood, R. Rannacher, Finite-element approximation of the nonstationary Navier-Stokes problem. IV. Error analysis for second-order time discretization, SIAM J. Numer. Anal., 27 (1990), 353-384.

[5] Y. He, K. Li, Convergence and stability of finite element nonlinear Galerkin method for the Navier-Stokes equations, Numer. Math., 79 (1998), 77-106. 
[6] E. Süli, Convergence and nonlinear stability of the Lagrange-Galerkin method for the NavierStokes equations, Numer. Math., 53 (1988), 459-483.

[7] Y. Achdou, J.L. Guermond, Convergence analysis of a finite element projection/Lagrange-Galerkin method for the incompressible Navier-Stokes equations, SIAM J. Numer. Anal., 37 (2000), 799826.

[8] O. Pironneau, On the transport-diffusion algorithm and its applications to the Navier-Stokes equations, Numer. Math., 38 (1982), 309-332.

[9] J. Shen, On error estimates of some higher order projection and penalty-projection methods for Navier-Stokes equations, Numer. Math., 62 (1992), 49-73.

[10] Z. Chen, K. Hoffmann, Numerical studies of a non-stationary Ginzburg-Landau model for the superconductivity, Adv. Math. Sci. Appl., 5 (1995), 363-389.

[11] J. Douglas Jr, R. Ewing, M. Wheeler, A time-discretization procedure for a mixed finite element approximation of miscible displacement in porous media, RAIRO Anal. Numer., 17 (1983), 249265.

[12] G. Akrivis, S. Larsson, Linearly implicit finite element methods for the time dependent Joule heatuing problem, BIT Numer. Anal., 45 (2005), 429-442.

[13] C. Elliott, S. Larsson, A finite element model for the time-dependent Joule heating problem, Math. Comput., 64 (1995), 1433-1453.

[14] Y. Tourigny, Optimal $H^{1}$ estimates for two time-discrete Galerkin approximations fof a nonlinear Schrödinger equation, IMA J. Numer. Anal., 11 (1991), 509-523.

[15] B. Li, W. Sun, Error analysis of linearized semi-implicit Galerkin finite element methods for nonlinear parabolic equations, Int. J. Numer. Anal. Model., 10 (2013), 622-633.

[16] B. Li, W. Sun, Unconditional convergence and optimal error estimates of a Galerkin-mixed FEM for incompressible miscible flow in porous media, SIAM J. Numer. Anal., 51 (2013), 1959-1977.

[17] H. Gao, Optimal error analysis of Galerkin FEMs for nolinear Joule heating equations, J. Sci. Comput., 58 (2014), 627-647.

[18] H. Gao, B. Li, W. Sun, Optimal error estimates of linearized Crank-Nicolson Galerkin FEMs for the time-dependent Ginzburg-Landau equations in superconductivity, SIAM J. Numer. Anal., 52 (2014), 1183-1202.

[19] H. Gao, Optimal error estimates of a linearized backward Euler FEM for the Landau-Lifshitz equation, SIAM J. Numer. Anal., 52 (2014), 2574-2593.

[20] D. Li, J. Wang, J. Zhang, Unconditionally convergent L1-Galerkin FEMs for nonlinear timefractional Schrödinger equations, SIAM J. Sci. Comput., 39 (2017), A3067-A3088.

[21] D. Shi, J. Wang, F. Yan, Unconditional superconvergence analysis for nonlinear parabolic equation with $E Q_{1}^{\text {rot }}$ nonconforming finite element, J. Sci. Comput., 70 (2017), 85-111.

[22] D. Shi, J. Wang, Unconditional superconvergence analysis of a Crank-Nicolson Galerkin FEM for nonlinear Schrödinger equation, J. Sci. Comput., 72 (2017), 1093-1118.

[23] Z. Si, J. Wang, W. Sun, Unconditional stability and error estimates of a modified characteristics FEMs for the Navier-Stokes equations, Numer. Math., 134 (2016), 139-161.

[24] J. Pan, Global superconvergence for the bilinear-constant scheme for the Stokes problem, SIAM J. Numer. Anal., 36 (1997), 2424-2430.

[25] H. Liu, N. Yan, Global superconvergence for optimal control problems governed by Stokes equations, Int. J. Numer. Anal. Model., 3 (2006), 283-302.

[26] R. Adams, Sobolev Spaces, Academics Press, 2003.

[27] R. Bermejo, P. Galan del Sastre, L. Saavedral, A second order in time modified Lagrange-Galerkin finite element method for the incompressible Navier-Stokes equations, SIAM J. Numer. Anal., 50 (2012), 3084-3109.

[28] J. Guaman, D. Leykekhman, Pointwise error estimates of finite element approximations to Stokes problems on convex polyhedra, Math. Comput., 81 (2012), 1879-1902. 